\title{
BERTINI THEOREMS FOR F-SIGNATURE AND HILBERT-KUNZ MULTIPLICITY
}

\author{
JAVIER CARVAJAL-ROJAS, KARL SCHWEDE, AND KEVIN TUCKER
}

\begin{abstract}
We show that Bertini theorems hold for $F$-signature and Hilbert-Kunz multiplicity. In particular, if $X \subseteq \mathbb{P}^{n}$ is normal and quasi-projective with $F$-signature greater than $\lambda$ (respectively the Hilbert-Kunz multiplicity is less than $\lambda$ ) at all points $x \in X$, then for a general hyperplane $H \subseteq \mathbb{P}^{n}$ the $F$-signature (respectively Hilbert-Kunz multiplicity) of $X \cap H$ is greater than $\lambda$ (respectively less than $\lambda$ ) at all points $x \in X \cap H$.
\end{abstract}

\section{INTRODUCTION}

A common tool for studying a quasi-projective algebraic variety $X \subseteq \mathbb{P}_{k}^{n}, k=\bar{k}$, is to perform induction on dimension by intersecting with a general hyperplane $H$. When doing this, we want the resulting intersection $X \cap H$ to have similar properties to the original variety $X$. Bertini's theorem accomplishes exactly this: the classical result asserts that if $X$ is smooth then so is $X \cap H$ for a general choice of $H$ [Har77, II, Theorem 8.18], [Kle98]. Many classes of singularities also satisfy this property. For example, in characteristic zero, if $X$ is $\log$ terminal (respectively $\log$ canonical), then so is $X \cap H$ [KM98, Lemma 5.17]. Even more generally the multiplier ideal of a divisor pair restricts to the multiplier ideal of the intersection

$$
\left.\mathcal{J}(X, \Delta)\right|_{X \cap H}=\mathcal{J}\left(X \cap H, \Delta_{X \cap H}\right),
$$

see [Laz04, Example 9.5.9]. In characteristic zero, Bertini theorems can be generalized to the case where $H$ is a general member of a base point free linear system.

In characteristic $p>0$, the situation is more complicated. It is essential that $H$ is a general member of a very ample linear system (or something close to that) if you expect Bertini-type results to hold. Since strongly $F$-regular and $F$-pure singularities are analogous to log terminal and log canonical singularities respectively [HW02], it is natural to expect that the corresponding Bertini-results hold. In [SZ13], this is exactly what was shown.

Theorem ([SZ13]). If $(X, \Delta)$ is a strongly F-regular (resp. sharply F-pure) pair such that $X \subseteq \mathbb{P}_{k}^{n}$ is quasi-projective and $k=\bar{k}$ is of characteristic $p>0$, then $\left(X \cap H,\left.\Delta\right|_{X \cap H}\right)$ is also strongly F-regular (resp. sharply F-pure) for a general choice of hyperplane $H \subseteq \mathbb{P}_{k}^{n}$.

However, the corresponding result for test ideals is false:

The first named author was supported in part by the NSF FRG Grant DMS \#1265261/1501115 and NSF CAREER Grant DMS \#1252860/1501102 and by the ERC-STG \#804334.

The second named author was supported in part by the NSF FRG Grant DMS \#1265261/1501115, NSF CAREER Grant DMS \#1252860/1501102 and NSF Grant DMS \#1801849.

The third named author was supported in part by NSF Grants DMS \#1602070 and \#1707661 and a fellowship from the Sloan foundation. 
Theorem $([\mathrm{Byd} 18])$. For any $p>0$ and $n \geq 3$, there exists a $\mathbb{Q}$-divisor $\Delta$ on $X=\mathbb{A}_{k}^{n}$, where $k=\bar{k}$ is of characteristic $p>0$, such that

$$
\left.\tau(X, \Delta)\right|_{H} \neq \tau\left(X \cap H,\left.\Delta\right|_{X \cap H}\right)
$$

for a general hyperplane $H \subseteq \mathbb{A}^{n}$.

It is then natural to ask about other types of $F$-singularities in characteristic $p>0$. For example, the behavior of $F$-rational singularities under restriction to general hyperplanes is still unknown. In this paper, we show that the above sort of Bertini-theorem holds for $F$-signature $s\left(\mathscr{O}_{X, x}\right)$ and Hilbert-Kunz multiplicity $e_{\mathrm{HK}}\left(\mathscr{O}_{X, x}\right)$ in the following sense.

Main Theorem (Theorem 5.4, Theorem 5.5). Suppose that $X \subseteq \mathbb{P}_{k}^{n}$ is a normal quasiprojective variety, $k=\bar{k}$ is of characteristic $p>0$, and $\Delta \geq 0$ is a $\mathbb{Q}$-divisor. Suppose that $\lambda \geq 0$ is a number such that the $F$-signature is bigger than $\lambda$,

$$
s\left(\mathscr{O}_{X, x}, \Delta\right)>\lambda,
$$

for all $x \in X$. Then for a general hyperplane $H \subseteq \mathbb{P}_{k}^{n}$,

$$
s\left(\mathscr{O}_{X \cap H, x},\left.\Delta\right|_{X \cap H}\right)>\lambda
$$

for all $x \in X \cap H$.

Similarly suppose that $\lambda \geq 1$ is a number such that the Hilbert-Kunz multiplicity is less than $\lambda$,

$$
e_{\mathrm{HK}}\left(\mathscr{O}_{X, x}\right)<\lambda,
$$

for all $x \in X$. Then for a general hyperplane $H \subseteq \mathbb{P}_{k}^{n}$,

$$
e_{\mathrm{HK}}\left(\mathscr{O}_{X \cap H, x}\right)<\lambda
$$

for all $x \in X \cap H$.

We actually prove a slightly stronger result by weakening the hypothesis that $X \subseteq \mathbb{P}_{k}^{n}$ and we also make statements about the locus $U$ where $s\left(\mathscr{O}_{X, x}, \Delta\right)>\lambda$ for all $x \in U$ or likewise with the locus where $e_{\mathrm{HK}}\left(\mathscr{O}_{X, x}\right)<\lambda$.

Recall that $F$-signature measures how strongly $F$-regular a variety or pair is. Explicitly, if $R$ is finite type over $k=\bar{k}$, then $R$ is regular if and only if $R^{1 / p^{e}}$ is a locally free $R$ module by [Kun69]. The $F$-signature refines this. By definition, $s(R)$ is a number that indicates what percentage of $R^{1 / p^{e}}$ is locally free asymptotically as $e$ goes to $\infty$. Thus $1 \geq s(R) \geq 0$ and

$\circ s(R)=1$ if and only if $R$ is regular [HL02 (cf. YYao05) and

$\circ s(R)>0$ if and only if $R$ is strongly $F$-regular [AL03].

The $F$-signature should be thought of some sort of local volume of the singularity.

On the other hand, Hilbert-Kunz measures how close a ring is to being regular. If $\left(R, \mathfrak{m}, k=k^{p}\right)$ is a local ring of dimension $d$, then $e_{\mathrm{HK}}(R)$ is the asymptotic value of the ratio between the number of generators of $R^{1 / p^{e}}$ as an $R$-module with the number of generators expected for a regular ring $\left(p^{e d}\right)$. One has that $e_{\mathrm{HK}}(R) \geq 1$ and

$\circ e_{\mathrm{HK}}(R)=1$ if and only if $R$ is regular [WY00, Theorem 1.5].

Hilbert-Kunz multiplicity is another sort of volume of a singularity.

We prove our main result by relying on the axiomatic Bertini framework as introduced in CGM86. In particular, to show the type of result in our Main Theorem, it suffices to show the following two properties for a property of singularities $\mathscr{P}\left(\operatorname{such}\right.$ as $\left.s\left(\mathscr{O}_{X, x}\right)>\lambda\right)$ : 
(A1) If $\phi: Y \rightarrow Z$ is a flat morphism with regular fibers and $Z$ is $\mathscr{P}$, then $Y$ is $\mathscr{P}$ too. (A2) Let $\phi: Y \rightarrow S$ be a morphism of finite type where $Y$ is excellent and $S$ is integral with generic point $\eta$. If $Y_{\eta}$ is geometrically $\mathscr{P}$, then there exists an open neighborhood $U$ of $\eta$ in $S$ such that the fibers $Y_{s}$ are geometrically $\mathscr{P}$ for each $s \in U$. (In fact, it suffices to check this for $S=\left(\mathbb{P}_{k}^{n}\right)^{*}$, the space of hyperplanes).

Property (A1) was already proven for $F$-signature in Yao06. In Section 3, we generalize this result to the context of pairs and give a new proof in the classical non-pair setting. In Section 4, we show that property (A2) holds for $F$-signature.

Acknowledgements: The authors thank Patrick Graf and Yongwei Yao for stimulating discussions. Work on this project was conducted in CIRM (Luminy) and Oberwolfach. They also thank the anonymous referee for very valuable comments and suggestions.

\section{Preliminaries}

2.1. Hilbert-Kunz multiplicity and $F$-signature. Throughout this article, we shall assume all schemes $X$ are Noetherian, separated, and have prime characteristic $p>0$. If $x \in X$, we let $k(x)$ denote the residue field of the local ring $\mathscr{O}_{X, x}$. We let $F^{e}: X \rightarrow X$ denote the $e$-iterated Frobenius endomorphism or $p^{e}$-th power map. We say $X$ is $F$-finite if $F^{e}$ is a finite morphism, in which case $X$ is automatically excellent and has a dualizing complex Kun76, Gab04].

When $X=\operatorname{Spec}(A)$ is affine, we often conflate scheme-theoretic and ring-theoretic notation. In particular, $F^{e}: A \rightarrow A$ denotes the $e$-iterated Frobenius, and for an $A$ module $M$ we write $F_{*}^{e} M$ for $\Gamma\left(\operatorname{Spec}(A), F_{*}^{e} \widetilde{M}\right)$ where $\widetilde{M}$ is the associated quasi-coherent sheaf on $\operatorname{Spec}(A)$. In other words, $F_{*}^{e} M$ is the $A$-module arising from $M$ via restriction of scalars for $F^{e}$. In case $A$ is reduced, we also identify $F^{e}$ with the inclusion $A \subseteq A^{1 / p^{e}}$, and shall at times use $M^{1 / p^{e}}$ to denote $F_{*}^{e} M$ accordingly.

If $J \subseteq A$ is an ideal, then the $e$-th Frobenius power of $J$ is the expansion of $J$ under the $e$-iterated Frobenius and denoted $J^{\left[p^{e}\right]}=\left\langle F^{e}(J)\right\rangle=\left\langle j^{\left[p^{e}\right]} \mid j \in J\right\rangle$. It follows $J\left(F_{*}^{e} M\right)=F_{*}^{e}\left(J^{\left[p^{e}\right]} M\right)$ or $J\left(M^{1 / p^{e}}\right)=\left(J^{\left[p^{e}\right]} M\right)^{1 / p^{e}}$ for any $A$-module $M$. In the local setting, the Frobenius powers give rise to the following well-studied variant on the HilbertSamuel multiplicity.

Definition 2.1. If $(A, \mathfrak{m})$ is a local ring of dimension $d$, the Hilbert-Kunz multiplicity of $A$ is

$$
e_{\mathrm{HK}}(A)=\lim _{e \rightarrow \infty} \frac{1}{p^{e d}} \ell_{A}\left(A / \mathfrak{m}^{\left[p^{e}\right]}\right),
$$

where we write $\ell_{A}\left(\_\right)$for the length of an $A$-module

Theorem 2.2. Suppose $(A, \mathfrak{m})$ is a local ring of dimension $d$.

(a) Mon83 The limit defining the Hilbert-Kunz multiplicity $e_{\mathrm{HK}}(A)$ exists, and moreover

$$
\ell_{A}\left(A / \mathfrak{m}^{\left[p^{e}\right]}\right)=e_{\mathrm{HK}}(A) \cdot p^{e d}+O\left(p^{e(d-1)}\right) .
$$

(b) WY00] The Hilbert-Kunz multiplicity $e_{\mathrm{HK}}(A) \geq 1$, and if $A$ is equidimensional then $e_{\mathrm{HK}}(A)=1$ if and only if $A$ is regular.

The $F$-signature, like the Hilbert-Kunz multiplicity, is another important numerical invariant of a local ring in positive characteristic defined in terms of the iterates of Frobenius. For any positive characteristic ring $A$, recall that an $A$-module inclusion $M_{1} \rightarrow M_{2}$ 
is said to be pure if $M_{1} \otimes_{A} N \rightarrow M_{2} \otimes_{A} N$ remains injective for any $A$-module $N$. An inclusion $A \rightarrow M$, where $M$ is a finitely generated $A$-module, is pure if and only if it is split, i.e. admits an $A$-module section; see [HR76, Corollary 5.2]. If $(A, \mathfrak{m})$ is local, $A \rightarrow M$ is pure if and only if $E_{A}(k) \rightarrow M \otimes_{A} E_{A}(k)$ is injective, where $E_{A}(k)$ is an injective hull of the residue field $k=A / \mathfrak{m}$; this follows from Matlis duality [HH95, Lemma 2.1 (e)]. We write $\ell_{A}\left(\_\right)$for the length of an $A$-module, omitting the subscript at times to simplify notation.

Definition 2.3. If $(A, \mathfrak{m})$ is an excellent local ring of dimension $d$, the $e$-th Frobenius degeneracy ideal

$$
\left.I_{e}(A)=\langle a \in A| A \stackrel{1 \mapsto F_{*}^{e} a}{\longrightarrow} F_{*}^{e} A \text { is not a pure } A \text {-module inclusion }\right\rangle
$$

is an ideal of $A$, and the $F$-signature is

$$
s(A)=\lim _{e \rightarrow \infty} \frac{1}{p^{e d}} \ell_{A}\left(A / I_{e}(A)\right) .
$$

Recall the following results on $F$-signature.

Theorem 2.4. Suppose $(A, \mathfrak{m})$ is an excellent local ring of dimension $d$.

(a) Tuc12] The limit defining the $F$-signature $s(A)$ exists, and moreover

$$
\ell_{A}\left(A / I_{e}(A)\right)=s(A) \cdot p^{e d}+O\left(p^{e(d-1)}\right) .
$$

(b) HL02 The $F$-signature $s(A) \leq 1$, and $s(A)=1$ if and only if $A$ is regular.

(c) [AL03, Yao05] The $F$-signature $s(A) \geq 0$, and $s(A)>0$ if and only if $A$ is strongly $F$-regular. In this case, $A$ is necessarily a Cohen-Macaulay normal domain.

The Hilbert-Kunz multiplicity and $F$-signature are also known to satisfy additional properties in the $F$-finite setting, such as semi-continuity.

Theorem 2.5. Smi16, Pol18, PT18] Consider an F-finite domain A.1]

(a) The Hilbert-Kunz mulitiplicity determines an upper semi-continuous function

$$
Q \in \operatorname{Spec}(A) \mapsto e_{\mathrm{HK}}\left(A_{Q}\right)
$$

on $\operatorname{Spec}(A)$.

(b) The F-signature determines a lower semi-continuous function

$$
Q \in \operatorname{Spec}(A) \mapsto s\left(A_{Q}\right)
$$

on $\operatorname{Spec}(A)$.

Moreover, if $(A, \mathfrak{m})$ is an $F$-finite local ring of dimension $d$, note that one can alternately describe the degeneracy ideals as

$$
\begin{aligned}
I_{e}(A) & \left.=\langle a \in A| A \stackrel{1 \mapsto F_{*}^{e} a}{\longrightarrow} F_{*}^{e} A \text { is not a split } A \text {-module inclusion }\right\rangle \\
& \left.=\langle a \in A| \phi\left(F_{*}^{e} a\right) \in \mathfrak{m} \text { for all } \phi \in \operatorname{Hom}_{A}\left(F_{*}^{e} A, A\right)\right\rangle,
\end{aligned}
$$

\footnotetext{
${ }^{1}$ Note that upper semi-continuiuty of the Hilbert-Kunz multiplicity is also known to hold for a ring which is essentially of finite type over an excellent local ring.
} 
and the $F$-signature can be viewed as giving an asymptotic measure of the number of splittings of the $e$-iterated Frobenius. In particular, if $(A, \mathfrak{m})$ is an $F$-finite local domain, we have

$$
e_{\mathrm{HK}}(A)=\lim _{e \rightarrow \infty} \frac{\mu_{A}\left(A^{1 / p^{e}}\right)}{\operatorname{rank}_{A}\left(A^{1 / p^{e}}\right)} \quad \text { and } \quad s(A)=\lim _{e \rightarrow \infty} \frac{\operatorname{frk}_{A}\left(A^{1 / p^{e}}\right)}{\operatorname{rank}_{A}\left(A^{1 / p^{e}}\right)} .
$$

where $\mu_{A}\left(\_\right)$denotes the minimal number of generators and $\operatorname{frk}_{A}\left(\_\right)$denotes free rank; see [PT18] for details. Recall that, for arbitrary (and not necessarily local) $A$, the free rank of an $A$-module $M$ is the maximal $\operatorname{rank} \operatorname{frk}_{A}(M)$ of a free $A$-module quotient of $M$. For us going forward, we will use $a_{e}(R)$ (resp. $\left.b_{e}(R)\right)$ to denote the free rank (resp. minimal number of generators) of $R^{1 / p^{e}}$, and may write simply $a_{e}\left(r e s p . b_{e}\right)$ if the context is clear

One can generalize the interpretation of Hilbert-Kunz multiplicity and $F$-signature for $F$-finite rings beyond the local setting as well. To make this more precise, recall first the following result of Kunz.

Lemma 2.6. [Kun76] If $A$ is a reduced equidimensional $F$-finite ring, the function

$$
Q \in \operatorname{Spec}(A) \mapsto\left[k(Q)^{1 / p^{e}}: k(Q)\right] \cdot p^{e \text { ht } Q}
$$

is constant on $\operatorname{Spec}(A)$. In particular, if $A$ is a domain,

$$
\operatorname{rank}_{A}\left(A^{1 / p^{e}}\right)=\left[k(Q)^{1 / p^{e}}: k(Q)\right] \cdot p^{e \text { ht } Q}
$$

for any $e \geq 0$ and $Q \in \operatorname{Spec}(A)$.

We recall a recent result globalizing Hilbert-Kunz multiplicity and F-signature.

Theorem 2.7. [DSPY19] If $A$ is a reduced equidimensional $F$-finite ring, and $\gamma \in \mathbb{Z}_{\geq 0}$ with $p^{\gamma}=\left[k(Q)^{1 / p}: k(Q)\right] \cdot p^{\text {ht } Q}$ for all $Q \in \operatorname{Spec}(A)$, then the limit

$$
e_{\mathrm{HK}}(A)=\lim _{e \rightarrow \infty} \frac{\mu_{A}\left(A^{1 / p^{e}}\right)}{p^{e \gamma}}
$$

exists and equals $\max \left\{e_{\mathrm{HK}}\left(A_{Q}\right) \mid Q \in \operatorname{Spec}(A)\right\}=\max \left\{e_{\mathrm{HK}}\left(A_{\mathfrak{m}}\right) \mid \mathfrak{m} \in \max \operatorname{Spec}(A)\right\}$. Similarly, the limit

$$
s(A)=\lim _{e \rightarrow \infty} \frac{\operatorname{frk}_{A}\left(A^{1 / p^{e}}\right)}{p^{e \gamma}}
$$

exists and equals $\min \left\{s\left(A_{Q}\right) \mid Q \in \operatorname{Spec}(A)\right\}=\min \left\{s\left(A_{\mathfrak{m}}\right) \mid \mathfrak{m} \in \max \operatorname{Spec}(A)\right\}$.

2.2. Divisors. In this subsection, we review the definitions and properties of the $F$ signature of divisor pairs.

Definition 2.8. If $(A, \mathfrak{m})$ is a normal excellent local domain of dimension $d$ and $D$ is an effective Weil divisor on $\operatorname{Spec}(A)$, the $e$-th Frobenius degeneracy ideal along $D$ is

$$
\left.I_{e}(A, D)=\langle a \in A| A \stackrel{1 \mapsto F_{*}^{e} a}{\longrightarrow} F_{*}^{e}(A(D)) \text { is not a pure } A \text {-module inclusion }\right\rangle .
$$

If $\Delta$ is an effective $\mathbb{Q}$-divisor on $\operatorname{Spec}(A)$, the $F$-signature of $(A, \Delta)$ is

$$
s(A, \Delta)=\lim _{e \rightarrow \infty} \frac{1}{p^{e d}} \ell_{A}\left(A / I_{e}\left(A,\left\lceil\left(p^{e}-1\right) \Delta\right\rceil\right)\right) .
$$


Lemma 2.9. Suppose $(A, \mathfrak{m})$ is a normal excellent local domain of dimension d and $\Delta$ is an effective $\mathbb{Q}$-divisor on $\operatorname{Spec}(A)$. Let $\left\{D_{e}\right\}_{e}>0$ be a sequence of Weil divisors on $\operatorname{Spec}(A)$ with bounded difference from $\left\{\left\lceil\left(p^{e}-1\right) \Delta\right\rceil\right\}_{e>0}$ independent of $e>0$. In other words, there exists an effective Cartier divisor $C$ such that

$$
-C \leq D_{e}-\left\lceil\left(p^{e}-1\right) \Delta\right\rceil \leq C
$$

for all $e>0$. Then

$$
s(A, \Delta)=\lim _{e \rightarrow \infty} \frac{1}{p^{e d}} \ell_{A}\left(A / I_{e}\left(A, D_{e}\right)\right) .
$$

Proof. This is essentially the same argument as [BST12, Lemma 4.17] and [PT18, Theorem 4.13], and so we omit it.

Theorem 2.10. BST12, PT18 Suppose $(A, \mathfrak{m})$ is a normal excellent local domain of dimension $d$ and $\Delta$ is an effective $\mathbb{Q}$-divisor on $\operatorname{Spec}(A)$.

(a) The limit defining the $F$-signature $s(A, \Delta)$ exists, and moreover

$$
\ell_{A}\left(A / I_{e}\left(A,\left\lceil\left(p^{e}-1\right) \Delta\right\rceil\right)\right)=s(A, \Delta) \cdot p^{e d}+O\left(p^{e(d-1)}\right) .
$$

(b) The $F$-signature $s(A, \Delta) \geq 0$, and $s(A, \Delta)>0$ if and only if $(A, \Delta)$ is strongly F-regular.

If $A$ is an $F$-finite normal excellent domain of dimension $d$ and $D$ is an effective Weil divisor on $\operatorname{Spec}(A)$, one can define the free rank of $A^{1 / p^{e}}$ along $D$

$$
a_{e}^{D}(A)=\operatorname{frk}_{A}^{D}\left(A^{1 / p^{e}}\right)
$$

to be the maximal rank $a_{e}(D)$ of a simultaneous free $A$-module quotient of $A^{1 / p^{e}}$ and $(A(D))^{1 / p^{e}}$. In other words, $a_{e}(D)$ is the largest non-negative integer such that there is a commuting diagram

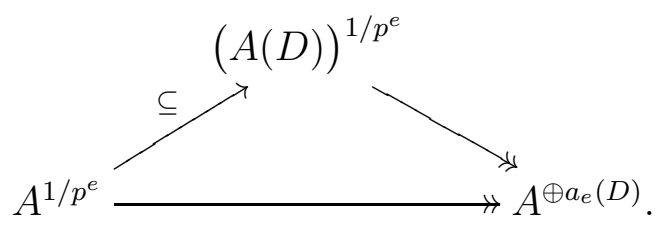

In case $(A, \mathfrak{m})$ is local, we have that $\operatorname{frk}_{A}^{D}\left(A^{1 / p^{e}}\right)=\left[k(\mathfrak{m})^{1 / p^{e}}: k(\mathfrak{m})\right] \cdot \ell_{A}\left(A / I_{e}(A, D)\right)$ (see [BST12, Proposition 3.5]), and once more this leads to a recent global interpretation of the $F$-signature along a divisor.

Theorem 2.11. [DSPY19] Let $A$ be an F-finite normal excellent domain of dimension $d$, and $\gamma \in \mathbb{Z}_{\geq 0}$ with $p^{\gamma}=\left[k(Q)^{1 / p}: k(Q)\right] \cdot p^{\mathrm{ht} Q}$ for all $Q \in \operatorname{Spec}(A)$. Suppose $\Delta$ is an effective $\mathbb{Q}$-divisor on $\operatorname{Spec}(A)$. The $F$-signature along $\Delta$ determines a lower semicontinuous function

$$
Q \in \operatorname{Spec}(A) \mapsto s\left(A_{Q}, \Delta\right)
$$

on $\operatorname{Spec}(A)$. Moreover, the limit

$$
s(A, \Delta)=\lim _{e \rightarrow \infty} \frac{\operatorname{frk}_{A}^{\left[p^{e} \Delta\right]}\left(A^{1 / p^{e}}\right)}{p^{e \gamma}}
$$

exists and equals $\min \left\{s\left(A_{Q}, \Delta\right) \mid Q \in \operatorname{Spec}(A)\right\}=\min \left\{s\left(A_{\mathfrak{m}}, \Delta\right) \mid \mathfrak{m} \in \max \operatorname{Spec}(A)\right\}$. 
In light of Theorem 2.11, and following [DSPY19], we also make the following global definition.

Definition 2.12. For a normal $F$-finite scheme $X$ and effective $\mathbb{Q}$-divisor $\Delta$ we set

$$
s(X, \Delta)=\min \left\{s\left(\mathscr{O}_{X, x}, \Delta\right) \mid x \in X\right\}=\min \left\{s\left(\mathscr{O}_{X, x}, \Delta\right) \mid x \in X \text { a closed point }\right\} .
$$

When $X=\operatorname{Spec} A$ is affine, we write $s(A, \Delta)$ for $s(X, \Delta)$.

2.3. Divisors and families. Finally, we discuss the correspondence between $\mathbb{Q}$-divisors and $p^{-e}$-linear maps in the relative setting of $A \subseteq R$ (or in other words, for families). What follows is contained in [PSZ18] although we work in a less general setting.

Setting 2.13. Suppose that $A$ is an $F$-finite regular domain and suppose we have $A \subseteq R$ a flat finite type extension of rings with geometrically ${ }^{2}$ normal fibers. Additionally assume that for some choice of $\omega_{A}$,

$$
F^{!} \omega_{A} \cong \omega_{A}
$$

This always holds for rings essentially of finite type over a Gorenstein semi-local ring.

For any $A$-algebra $B$, we write $R_{B}=R \otimes_{A} B$. Frequent values of $B$ include $A^{1 / p^{e}}$, the fraction field $K:=K(A)$ and $k(Q)$, the residue field of a point $Q \in \operatorname{Spec} A$.

We make some quick observations.

Lemma 2.14. In the setting of Setting 2.13, each $R_{A^{1 / p^{e}}}$ is a normal integral domain, as are $R_{K^{1 / p^{e}}}$ and $R_{K^{\infty}}$ as well.

Proof. $A^{1 / p^{e}} \rightarrow R_{A^{1 / p^{e}}}$ is flat with normal fibers over a regular base, and hence $R_{A^{1 / p^{e}}}$ is normal by [Mat89, Theorem 23.9]. Since $R \rightarrow R_{A^{1 / p^{e}}}$ is purely inseparable and $R_{A^{1 / p^{e}}}$ is reduced, it follows that $R_{A^{1 / p^{e}}}$ is a domain. Localizing, we have that $K \rightarrow R_{K}$ also has geometrically normal fibers, and the same argument gives that $R_{K^{1 / p^{e}}}$ and $R_{K^{\infty}}$ are normal domains as well.

Lemma 2.15. In the setting of Setting 2.13, for each $Q \in \operatorname{Spec} A$ and $x \in \operatorname{Spec} R_{K(Q)} \subseteq$ Spec $R$ a point of codimension 1 on the fiber, we have that $R_{x}$ is regular and thus $\Delta$ is $\mathbb{Q}$-Cartier at $x$. In particular, we can restrict $\left.\Delta\right|_{\operatorname{Spec} R_{k(Q)}}$ to any fiber.

Proof. Choose a codimension 1 point $x \in \operatorname{Spec} R_{K(Q)}$, in other words a codimension one point of a fiber over $Q \in \operatorname{Spec}(A)$. In particular, $\left(R_{K(Q)}\right)_{x}$ is normal and hence regular. It follows that $R_{x}$ is also regular since $R_{K(Q)}$ is obtained from $R$ by killing a regular sequence and localizing.

We now discuss the correspondence between divisors and maps in Setting 4.1.

Lemma 2.16. [PSZ18, 2.8-2.11] Suppose that $A$ is an F-finite regular domain and suppose we have $A \subseteq R$ a flat finite type extension of rings with geometrically normal fibers. Then for every $R_{A^{1 / p^{e}} \text {-linear map }}$

$$
\phi: R^{1 / p^{e}} \rightarrow R_{A^{1 / p^{e}}}
$$

which generates $\operatorname{Hom}_{R_{A^{1 / p}}}\left(R^{1 / p^{e}}, R_{A^{1 / p^{e}}}\right)$ at the generic point of every fiber, there exists a corresponding $\mathbb{Z}_{(p)}$-divisol $\sqrt{3}$ on $\operatorname{Spec} R$

$$
\Delta_{\phi} \sim_{\mathbb{Q}}-K_{R / A}
$$

\footnotetext{
${ }^{2}$ Here we mean that the fibers are normal after any base change, including inseparable ones.

${ }^{3} \mathrm{~A} \mathbb{Q}$-divisor in which no denominators contain $p$.
} 
which does not contain any fiber in its support.

Conversely, given an effective $\mathbb{Z}_{(p)}$-divisor $\Delta \sim_{\mathbb{Q}}-K_{R / A}$ on $\operatorname{Spec} R$ whose support does not contain any fiber, we can construct a map $\phi: R^{1 / p^{e}} \rightarrow R_{A^{1 / p^{e}}}$ such that $\Delta_{\phi}=\Delta$.

Finally, we recall the interaction between divisors and maps behaves under base change. While not crucial for the following statement, in this paper we restrict ourselves to base changes which are either flat or restriction to a fiber followed by a flat base change, which is easier to work with than the generality of [PSZ18].

Lemma 2.17. [PSZ18, Lemma 2.21] In the setting of Setting 2.13 assume that $\Delta=\Delta_{\phi}$ is constructed as in Lemma 2.16. For any regular A-algebra $B$ satisfying (IT), let $\pi$ : Spec $R_{B} \rightarrow$ Spec $R$ denote the canonical map. Set $\phi_{B}:=\phi \otimes_{A^{1 / p^{e}}} B^{1 / p^{e}}$ to be the base changed map

$$
\phi_{B}:\left(R_{B}\right)^{1 / p^{e}}=R^{1 / p^{e}} \otimes_{A^{1 / p^{e}}} B^{1 / p^{e}} \rightarrow R_{A^{1 / p^{e}}} \otimes_{A^{1 / p^{e}}} B^{1 / p^{e}}=R_{B^{1 / p^{e}}} .
$$

In this case,

$$
\Delta_{\phi_{B}}=\pi^{*} \Delta=\pi^{*} \Delta_{\phi}
$$

Remark 2.18. Frequently $B=A^{1 / p^{d}}$ in which case the based changed map $\phi_{B}$ in Lemma 2.17 is simply

$$
\phi_{A^{1 / p^{d}}}:\left(R_{A^{1 / p^{d}}}\right)^{1 / p^{e}} \rightarrow R_{A^{1 / p^{e+d}}} .
$$

\section{F-Signature transformation FOR REGUlar FibERS}

In this section, we will be concerned with the behavior of the $F$-signature under flat local extensions, building on the following result of Y. Yao.

Theorem 3.1 ([Yao06]). Suppose that $(A, \mathfrak{m}) \subseteq(R, \mathfrak{n})$ is a flat local extension of excellent local rings of characteristic $p>0$. Then if $R / \mathfrak{m} R$ is regular, we have

$$
s(A)=s(R) .
$$

Our goal is to generalize the above result to the context of divisor pairs $(R, \Delta)$, for which we will first need to give a variation on the proof of the original statement. We begin with some preliminary lemmas.

Lemma 3.2. Suppose that $(A, \mathfrak{m}) \subseteq(R, \mathfrak{n})$ is a flat local extension of local rings. If $x_{1}, \ldots, x_{\delta} \in R$ is a regular sequence on $R / \mathfrak{m} R$, then $R /\left\langle x_{1}, \ldots, x_{\delta}\right\rangle$ is a flat A-algebra. Moreover, $x_{1}, \ldots, x_{\delta} \in R$ are a regular sequence on $M \otimes_{A} R$ for any finitely generated $A$-module $M$, and lastly for any $t \geq 0$ the $R$-module inclusion

$$
R /\left\langle x_{1}^{t}, \ldots, x_{\delta}^{t}\right\rangle \stackrel{1 \mapsto\left[\frac{1}{x_{1}^{t} \cdots x_{\delta}^{t}}\right]}{\longrightarrow} H_{\left\langle x_{1}, \ldots, x_{\delta}\right\rangle}^{\delta}(R)
$$

is pure as an inclusion of $A$-modules.

Proof. See [Mat80, Corollary 20.F, page 151] or [HH94, Lemma 7.10]. For the final statement, note that it suffices to check purity after tensoring with finitely generated $A$-modules, where injectivity follows from the previous regular sequence assertion.

The following was used in Hochster and Huneke's original study of $F$-regularity and base change. 
Lemma 3.3. [HH94, Lemma 7.10] Let $(A, \mathfrak{m}) \subseteq(R, \mathfrak{n})$ be a flat local extension of local rings. Suppose $R / \mathfrak{m} R$ is regular and $x_{1}, \ldots, x_{\delta} \in R$ give a regular system of parameters of $R / \mathfrak{m} R$. If $E_{A}$ is an injective hull of $A / \mathfrak{m}$ over $A$ with socle generated by $u$, then $E_{R}=H_{\left\langle x_{1}, \ldots, x_{\delta}\right\rangle}^{\delta}(R) \otimes_{A} E_{A}$ is an injective hull of $R / \mathfrak{n}$ over $R$ with socle generated by $\left[\frac{1}{x_{1} \cdots x_{\delta}}\right] \otimes u$.

Now, we give a new proof of Yao's result.

Proof of Theorem 3.1. If $x_{1}, \ldots, x_{\delta} \in R$ give a regular system of parameters of $R / \mathfrak{m} R$, then by Lemma 3.3 we have $E_{R}=H_{\left\langle x_{1}, \ldots, x_{\delta}\right\rangle}^{\delta}(R) \otimes_{A} E_{A}$ with socle generated by $v=$ $\left[\frac{1}{x_{1} \cdots x_{\delta}}\right] \otimes u$.

Consider now $R^{1 / p^{e}} \otimes_{R} E_{R}$, so that $I_{e}(R)^{1 / p^{e}}=\operatorname{Ann}_{R^{1 / p^{e}}}(1 \otimes v)$. With this observation in place, we claim the following equality of ideals of $R^{1 / p^{e}}$ :

Claim 3.4. $I_{e}(R)^{1 / p^{e}}=\left(I_{e}(A) R+\left\langle x_{1}^{p^{e}}, \ldots, x_{\delta}^{p^{e}}\right\rangle\right)^{1 / p^{e}}$

Proof of claim. We may identify $R^{1 / p^{e}} \otimes_{R} H_{\left\langle x_{1}, \ldots, x_{\delta}\right\rangle}^{\delta}(R)=\left(H_{\left\langle x_{1}, \ldots, x_{\delta}\right\rangle}^{\delta}(R)\right)^{1 / p^{e}}$ with $1 \otimes$ $\left[\frac{1}{x_{1} \cdots x_{\delta}}\right] \leftrightarrow\left[\frac{1}{x_{1}^{p^{e} \cdots x_{\delta}^{p^{e}}}}\right]^{1 / p^{e}}$. Using that

$$
\left(R /\left\langle x_{1}^{p^{e}}, \ldots, x_{\delta}^{p^{e}}\right\rangle\right)^{1 / p^{e}} \stackrel{1 \mapsto\left[\frac{1}{x_{1}^{p^{e} \cdots x_{\delta}^{p^{e}}}}\right]^{1 / p^{e}}}{\longrightarrow}\left(H_{\left\langle x_{1}, \ldots, x_{\delta}\right\rangle}^{\delta}(R)\right)^{1 / p^{e}}
$$

is pure as an inclusion of $A^{1 / p^{e}}$-modules, this gives further identifications

$$
\begin{array}{rlc}
R^{1 / p^{e}} \otimes_{R} E_{R} & = & \left(H_{\left\langle x_{1}, \ldots, x_{\delta}\right\rangle}^{\delta}(R)\right)^{1 / p^{e}} \otimes_{A^{1 / p^{e}}}\left(A^{1 / p^{e}} \otimes_{A} E_{A}\right) \\
1 \otimes v & \leftrightarrow & {\left[\frac{1}{x_{1}^{p^{e}} \cdots x_{\delta}^{p^{e}}}\right]^{1 / p^{e}} \otimes(1 \otimes u)} \\
& \supseteq & \left(R /\left\langle x_{1}^{p^{e}}, \ldots, x_{\delta}^{p^{e}}\right\rangle\right)^{1 / p^{e}} \otimes_{A^{1 / p^{e}}}\left(A^{1 / p^{e}} \otimes_{A} E_{A}\right) \\
& \leftrightarrow & 1 \otimes(1 \otimes u) .
\end{array}
$$

In particular, $\operatorname{Ann}_{R^{1 / p^{e}}}(1 \otimes v)=\operatorname{Ann}_{R^{1 / p^{e}}}(1 \otimes(1 \otimes u))$. It is worth noting that this ideal contains the kernel of $R^{1 / p^{e}} \rightarrow\left(R /\left\langle x_{1}^{p^{e}}, \ldots, x_{\delta}^{p^{e}}\right\rangle\right)^{1 / p^{e}}$ and its image along this quotient homomorphism is the same as the annihilator of $1 \otimes(1 \otimes u)$ over $\left(R /\left\langle x_{1}^{p^{e}}, \ldots, x_{\delta}^{p^{e}}\right\rangle\right)^{1 / p^{e}}$.

On the other hand, since $A^{1 / p^{e}} \rightarrow\left(R /\left\langle x_{1}^{p^{e}}, \ldots, x_{\delta}^{p^{e}}\right\rangle\right)^{1 / p^{e}}$ is flat, it follows that the annihilator of $1 \otimes(1 \otimes u)$ over $\left(R /\left\langle x_{1}^{p^{e}}, \ldots, x_{\delta}^{p^{e}}\right\rangle\right)^{1 / p^{e}}$ is the extension of

$$
\operatorname{Ann}_{A^{1 / p^{e}}}\left(1 \otimes u \in A^{1 / p^{e}} \otimes_{A} E_{A}\right)=I_{e}(A)^{1 / p^{e}} .
$$

Putting everything together proves the claim. 
Thus, using the flatness of $A \rightarrow R /\left\langle x_{1}^{p^{e}}, \ldots, x_{\delta}^{p^{e}}\right\rangle$ once again, it follows

$$
\begin{aligned}
\ell_{R}\left(\frac{R}{I_{e}(R)}\right) & =\ell_{R}\left(\frac{R}{I_{e}(A) R+\left\langle x_{1}^{p^{e}}, \ldots, x_{\delta}^{p^{e}}\right\rangle}\right)=\ell_{A}\left(\frac{A}{I_{e}(A)}\right) \ell_{R}\left(\frac{R}{\mathfrak{m} R+\left\langle x_{1}^{p^{e}}, \ldots, x_{\delta}^{p^{e}}\right\rangle}\right) \\
& =p^{e \delta} \ell_{A}\left(\frac{A}{I_{e}(A)}\right) \ell_{R}\left(\frac{R}{\mathfrak{m} R+\left\langle x_{1}, \ldots, x_{\delta}\right\rangle}\right)=p^{e \delta} \ell_{A}\left(\frac{A}{I_{e}(A)}\right) .
\end{aligned}
$$

Since $\operatorname{dim} R=\operatorname{dim} A+\delta$, the desired equality now follows after dividing by $p^{e \operatorname{dim} R}$ and taking limits.

We now generalize the above proof to the context of pairs. We break off the main technical step into a lemma.

Lemma 3.5. Suppose that $(A, \mathfrak{m}) \subseteq(R, \mathfrak{n})$ is a flat local extension of normal local rings of characteristic $p>0$ and write $f: \operatorname{Spec} R \rightarrow \operatorname{Spec} A$ for the induced map. For any effective Weil $D$ on $\operatorname{Spec} A$ and $e>0$, define

$$
\begin{aligned}
I_{e}(A, D) & \left.=\langle a \in A| A \rightarrow A(D)^{1 / p^{e}} \text { with } 1 \mapsto a^{1 / p^{e}} \text { is not A-pure }\right\rangle \\
I_{e}\left(R, f^{*} D\right) & \left.=\langle r \in R| R \rightarrow R\left(f^{*} D\right)^{1 / p^{e}} \text { with } 1 \mapsto r^{1 / p^{e}} \text { is not } R \text {-pure }\right\rangle .
\end{aligned}
$$

Then if $R / \mathfrak{m} R$ is regular,

$$
\ell_{R}\left(\frac{R}{I_{e}\left(R, f^{*} D\right)}\right)=p^{e(\operatorname{dim} R-\operatorname{dim} A)} \cdot \ell_{A}\left(\frac{A}{I_{e}(A, D)}\right) .
$$

Proof. If $x_{1}, \ldots, x_{\delta} \in R$ give a regular system of parameters of $R / \mathfrak{m} R$, we have that $E_{R}=H_{\left\langle x_{1}, \ldots, x_{\delta}\right\rangle}^{\delta}(R) \otimes_{A} E_{A}$ with socle generated by $v=\left[\frac{1}{x_{1} \cdots x_{\delta}}\right] \otimes u$. Consider now $R\left(f^{*} D\right)^{1 / p^{e}} \otimes_{R} E_{R}$, so that $I_{e}\left(R, f^{*} D\right)^{1 / p^{e}}=\operatorname{Ann}_{R^{1 / p^{e}}}(1 \otimes v)$. Using that $R\left(f^{*} D\right)=$ $R \otimes_{A} A(D)$ and the same identifications made in the proof above, we see that

$$
\begin{array}{rlc}
R\left(f^{*} D\right)^{1 / p^{e}} \otimes_{R} E_{R} & = & \left(H_{\left\langle x_{1}, \ldots, x_{\delta}\right\rangle}^{\delta}(R)\right)^{1 / p^{e}} \otimes_{A^{1 / p^{e}}}\left(A(D)^{1 / p^{e}} \otimes_{A} E_{A}\right) \\
1 \otimes v & \leftrightarrow & {\left[\frac{1}{x_{1}^{p^{e} \cdots x_{\delta}^{p^{e}}}}\right]^{1 / p^{e}} \otimes(1 \otimes u)} \\
& \supseteq & \left(R /\left\langle x_{1}^{p^{e}}, \ldots, x_{\delta}^{p^{e}}\right\rangle\right)^{1 / p^{e}} \otimes_{A^{1 / p^{e}}}\left(A(D)^{1 / p^{e}} \otimes_{A} E_{A}\right) \\
& \leftrightarrow & 1 \otimes(1 \otimes u) .
\end{array}
$$

But since $A^{1 / p^{e}} \rightarrow\left(R /\left\langle x_{1}^{p^{e}}, \ldots, x_{\delta}^{p^{e}}\right\rangle\right)^{1 / p^{e}}$ is flat, it follows that the annihilator of $1 \otimes(1 \otimes$ $u$ ) over $\left(R /\left\langle x_{1}^{p^{e}}, \ldots, x_{\delta}^{p^{e}}\right\rangle\right)^{1 / p^{e}}$ is the expansion of $\operatorname{Ann}_{A^{1 / p^{e}}}\left(1 \otimes u \in A(D)^{1 / p^{e}} \otimes_{A} E_{A}\right)=$ $I_{e}(A, D)^{1 / p^{e}}$. In other words, we have shown $I_{e}\left(R, f^{*} D\right)^{1 / p^{e}}=\left(I_{e}(A, D) R+\left\langle x_{1}^{p^{e}}, \ldots, x_{\delta}^{p^{e}}\right\rangle\right)^{1 / p^{e}}$. 
Thus, using the flatness of $A \rightarrow R /\left\langle x_{1}^{p^{e}}, \ldots, x_{\delta}^{p^{e}}\right\rangle$ once again, it follows

$$
\begin{aligned}
\ell_{R}\left(\frac{R}{I_{e}\left(R, f^{*} D\right)}\right) & =\ell_{R}\left(\frac{R}{I_{e}(A, D) R+\left\langle x_{1}^{p^{e}}, \ldots, x_{\delta}^{p^{e}}\right\rangle}\right) \\
& =\ell_{A}\left(\frac{A}{I_{e}(A, D)}\right) \ell_{R}\left(\frac{R}{\mathfrak{m} R+\left\langle x_{1}^{p^{e}}, \ldots, x_{\delta}^{p^{e}}\right\rangle}\right) \\
& =p^{e \delta} \ell_{A}\left(\frac{A}{I_{e}(A, D)}\right) \ell_{R}\left(\frac{R}{\mathfrak{m} R+\left\langle x_{1}, \ldots, x_{\delta}\right\rangle}\right)=p^{e \delta} \ell_{A}\left(\frac{A}{I_{e}(A, D)}\right)
\end{aligned}
$$

as desired.

We now can prove the main result of the section.

Theorem 3.6. Suppose that $(A, \mathfrak{m}) \subseteq(R, \mathfrak{n})$ is a flat local extension of normal local rings of characteristic $p>0$ and write $f: \operatorname{Spec} R \rightarrow \operatorname{Spec} A$ the induced map. Suppose further that $\Delta \geq 0$ is a $\mathbb{Q}$-divisor on $\operatorname{Spec} A$. Then if $R / \mathfrak{m} R$ is regular, we have

$$
s(A, \Delta, \mathfrak{m})=s\left(R, f^{*} \Delta, \mathfrak{n}\right) .
$$

Proof. We will first apply Lemma 3.5 to $D=\left\lfloor p^{e} \Delta\right\rfloor$. We see that $f^{*} D=f^{*}\left\lfloor p^{e} \Delta\right\rfloor \leq$ $\left\lfloor p^{e} f^{*} \Delta\right\rfloor$. Hence, recalling that $d=\operatorname{dim} R$ and applying both Lemma 2.9 and Lemma 3.5,

$$
\begin{aligned}
s\left(R, f^{*} \Delta\right) & =\lim _{e \rightarrow \infty} \frac{1}{p^{d e}} \ell_{R}\left(R / I_{e}\left(R,\left\lceil\left(p^{e}-1\right) f^{*} \Delta\right\rceil\right)\right)=\lim _{e \rightarrow \infty} \frac{1}{p^{d e}} \ell_{R}\left(R / I_{e}\left(R,\left\lfloor p^{e} f^{*} \Delta\right\rfloor\right)\right) \\
& \leq \lim _{e \rightarrow \infty} \frac{1}{p^{d e}} \cdot \ell_{R}\left(R / I_{e}\left(R, f^{*}\left\lfloor p^{e} \Delta\right\rfloor\right)\right)=\lim _{e \rightarrow \infty} \frac{1}{p^{(d-\delta) e}} \ell_{A}\left(A / I_{e}\left(A,\left\lfloor p^{e} \Delta\right\rfloor\right)\right) \\
& =s(A, \Delta) .
\end{aligned}
$$

On the other hand, if we choose $D=\left\lceil p^{e} \Delta\right\rceil$, then $f^{*} D=f^{*}\left\lceil p^{e} \Delta\right\rceil \geq\left\lceil p^{e} f^{*} \Delta\right\rceil$ and arguing as above gives $s\left(R, f^{*} \Delta\right) \geq s(A, \Delta)$. This completes the proof.

We also address Hilbert-Kunz multiplicity under flat extensions (with regular fibers). There is little work to do here since Kunz proved the result (even before a limit is taken).

Theorem 3.7. ([Kun76, Proposition 3.9b]) Let $(A, \mathfrak{m}) \hookrightarrow(R, \mathfrak{n})$ be a flat local extension of rings of positive characteristic. Further suppose that $R / \mathfrak{m} R$ is regular. Then

$$
e_{\mathrm{HK}}(A)=e_{\mathrm{HK}}(R) .
$$

\section{F-signature and Hilbert-Kunz multiplicity of General fibers}

Before proving Bertini-type theorems, we need one more result. We need to show that if $A \subseteq R$ is a finite type extension of rings such that the perfectified generic fiber has $F$-signature greater than $\lambda$, then so do most of the closed fibers.

Setting 4.1. We assume that $A \subseteq R$ is a flat finite type morphism of Noetherian $F$-finite integral domains with fraction fields $K=\operatorname{Frac}(A) \subseteq L=\operatorname{Frac}(R)$. Suppose further that $A$ is regular and that $A \subseteq R$ has geometrically normal fibers. Further assume that $\Delta \geq 0$ is a $\mathbb{Q}$-divisor on $\operatorname{Spec} R$ whose support does not contain any fiber.

We will not universally assume this setting in this section, but we will always be able to reduce to it. In order to motivate the main result of this section, we first give an easy proof of a weaker statement. 
Proposition 4.2. In the setting of Setting 4.1, further suppose that $A$ is finite type over an uncountable algebraically closed field of characteristic $p>0$. If

$$
s\left(R_{K^{\infty}, x}\right) \geq \lambda
$$

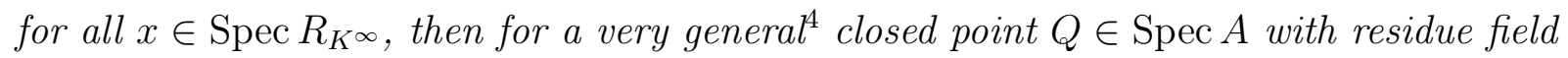
$k(Q)$,

for all $x \in \operatorname{Spec} R_{k(Q)}$.

$$
s\left(R_{k(Q), x}\right) \geq \lambda
$$

Proof. By [DSPY19, Theorem 4.13], for each $e>0$, and by Lemma 4.8 below, we can spread out our splitting and obtain some $a_{e}, d_{e}$ and $0 \neq g_{e} \in A$ so that there is a surjection

$$
R_{A\left[1 / g_{e}\right]^{1 / p^{e+d_{e}}}}^{1 / p^{e}} \rightarrow R_{A\left[1 / g_{e}\right]^{1 / p^{e+d_{e}}}}^{\oplus a_{e}}
$$

and so that

$$
\lambda \leq \min _{x \in \operatorname{Spec} R_{K^{\infty}}}\left\{s\left(R_{K^{\infty}, x}\right)\right\}=\lim _{e \rightarrow \infty} \frac{a_{e}}{p^{e \operatorname{dim} R}} .
$$

Since our $Q$ is very general, $Q \notin V\left(g_{e}\right)$ for any $e$. Hence we have surjections $A\left[1 / g_{e}\right] \rightarrow$ $k(Q)$ for all $e$. We now apply

to (4.2.1) which yields a surjective map

$$
\otimes_{A\left[1 / g_{e}\right]^{1 / p^{e+d_{e}}}} k(Q)^{1 / p^{e+d_{e}}}
$$

$$
R_{k(Q)^{1 / p^{e+d_{e}}}}^{1 / p^{e}} \rightarrow R_{k(Q)^{1 / p^{e+d_{e}}}}^{\oplus a_{e}}
$$

But $k(Q)$ is perfect and so this can be identified with a surjective map

$$
\left(R_{k(Q)}\right)^{1 / p^{e}} \rightarrow R_{k(Q)}^{\oplus a_{e}}
$$

The result follows.

Remark 4.3. We do not expect this result to hold for simply general fibers; see Mon98] for an example where the analagous Hilbert-Kunz statement for general fibers does not hold.

We now need the following result of Pérez, the third author, and Yao.

Theorem 4.4. PTY20] For every Noetherian ring A of characteristic $p>0$, and every finitely generated $A$-algebra $R$, and every finitely generated $R$-module $M$, there exists a positive constant $C$ with the following property: for all primes $Q \in \operatorname{Spec}(A)$, all regular $k(Q)$-algebras $\Gamma$, and all $P \in \operatorname{Spec}\left(R_{\Gamma}:=R \otimes_{A} \Gamma\right)$, and all $e \geq 1$, we have that $\ell_{R_{\Gamma}}\left(\left(M_{\Gamma}\right)_{P} / P^{\left[p^{e}\right]}\left(M_{\Gamma}\right)_{P}\right) \leq C p^{e \operatorname{dim}\left(M_{\Gamma}\right)}$ where $M_{\Gamma}:=M \otimes_{A} \Gamma$.

The next result is the technical heart of the section. We state and prove it first in the non-pairs setting and then explain how to generalize it to pairs in a proposition which follows it.

Notation 4.5. Recall again that if $S$ is a local ring, $a_{e}(S)$ denotes the free rank of $S^{1 / p^{e}}$ whereas $b_{e}(S)$ denotes its minimal number of generators. Moreover, if $\Delta$ is a $\mathbb{Q}$-divisor on Spec $S$ we shall denote by $a_{e}^{\Delta}(S)$ the number $a_{e}^{\left\lceil\left(p^{e}-1\right) \Delta\right\rceil}(S)$ defined in $(2.10 .1)$; which is a slight abuse of notation.

\footnotetext{
${ }^{4}$ Meaning outside a countable union of proper closed subsets of $\operatorname{Spec} A$
} 
Proposition 4.6. Suppose we are in the setting of Setting 4.1. There exists a positive constant $C$ and $0 \neq g \in A$ with the following property: for all $Q \in \operatorname{Spec}\left(B:=A\left[g^{-1}\right]\right)$, all $d>0$, all $x \in \operatorname{Spec}\left(R_{k(Q)^{1 / p^{d}}}\right)$, and all $e>0$, we have

$$
\left|s\left(R_{k(Q)^{1 / p^{d}, x}}\right)-\frac{a_{e}\left(R_{k(Q)^{1 / p^{d}, x}}\right)}{\operatorname{rank}_{R_{k(Q)^{1 / p^{d}}{ }_{, x}}}\left(R_{k(Q)^{1 / p^{d}, x}}\right)^{1 / p^{e}}}\right| \leq \frac{C}{p^{e}} .
$$

Similarly we have

$$
\left|e_{\mathrm{HK}}\left(R_{k(Q)^{1 / p^{d}, x}}\right)-\frac{b_{e}\left(R_{k(Q)^{1 / p^{d}, x}}\right)}{\operatorname{rank}_{R_{k(Q)^{1 / p^{d}, x}}}\left(R_{k(Q)^{1 / p^{d}, x}}\right)^{1 / p^{e}}}\right| \leq \frac{C}{p^{e}} .
$$

Proof. Let $\delta=\operatorname{dim} R_{K}=\operatorname{dim} R_{K^{\infty}}$, so that $\operatorname{rank}_{R_{K^{\infty}}}\left(R_{K^{\infty}}\right)^{1 / p^{e}}=p^{e \delta}$. Since $R_{A^{1 / p^{e+d}}} \rightarrow$ $R_{A^{1 / p^{e+d}}}^{1 / p^{e}}$ base changes to $R_{K^{\infty}} \rightarrow R_{K^{\infty}}^{1 / p^{e}}$ for any $e, d>0$, we see that

$$
\operatorname{rank}_{R} R_{A^{1 / p^{e+d}}} R_{A^{1 / p^{e+d}}}^{1 / p^{e}}=p^{e \delta}
$$

as well. Note that $A^{1 / p^{d}} \subseteq R_{A^{1 / p^{d}}}$ is also flat, and for any $Q \in \operatorname{Spec}(A)$ and $x \in$ $\operatorname{Spec}\left(R_{k(Q)^{1 / p^{d}}}\right)$, we have that ht $x-$ ht $Q=\operatorname{dim} R_{k(Q)^{1 / p^{d}}, x}$. Using that Frac $\left(R_{A^{1 / p^{e+d}}}\right)=$ $L_{K^{1 / p^{e+d}}}$ as $R_{A^{1 / p^{e+d}}}$ is a domain, we also compute

$$
\begin{aligned}
p^{e \delta}=\operatorname{rank}_{R_{A^{1 / p^{e}+d}}} R_{A^{1 / p^{e+d}}}^{1 / p^{e}}=\left[L_{K^{1 / p^{e+d}}}^{1 / p^{e}}: L_{K^{1 / p^{e+d}}}\right] & =\frac{\left[L_{K^{1 / p^{e+d}}}^{1 / p^{e}}: L_{K^{1 / p^{d}}}\right]}{\left[L_{K^{1 / p^{e+d}}}: L_{K^{1 / p^{d}}}\right]} \\
& =\frac{\left[L_{K^{1 / p^{e+d}}}^{1 / p^{e}}: L_{K^{1 / p^{d}}}\right]}{\left[K^{1 / p^{e}}: K\right]} \\
& =\frac{\left[k(x)^{1 / p^{e}}: k(x)\right]}{\left[k(Q)^{1 / p^{e}}: k(Q)\right]} \cdot p^{e(\mathrm{ht} x-\mathrm{ht} Q)} \\
& =\frac{\left[k(x)^{1 / p^{e}}: k(x)\right]}{\left[k(Q)^{1 / p^{e}}: k(Q)\right]} \cdot p^{e \operatorname{dim} R_{k(Q)^{1 / p^{d}},{ }_{, x}}}
\end{aligned}
$$

whence

$$
\left[k(Q)^{1 / p^{e}}: k(Q)\right] \cdot p^{e \delta}=\operatorname{rank}_{R_{k(Q)^{1 / p^{d}, x}}}\left(R_{k(Q)^{1 / p^{d}, x}}\right)^{1 / p^{e}} .
$$

Form exact sequences

$$
\begin{aligned}
& \left(R_{A^{1 / p}}\right)^{\oplus p^{\delta}} \stackrel{\alpha_{1}}{\rightarrow} R^{1 / p} \rightarrow M_{1} \rightarrow 0 \\
& R^{1 / p} \stackrel{\alpha_{2}}{\longrightarrow}\left(R_{A^{1 / p}}\right)^{\oplus p^{\delta}} \rightarrow M_{2} \rightarrow 0
\end{aligned}
$$

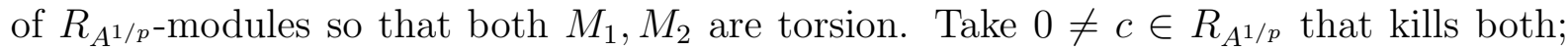
replacing $c$ with $c^{p}$ if necessary, we may further assume $0 \neq c \in R$. The image of $U=\operatorname{Spec} R[1 / c] \subseteq \operatorname{Spec} R$ in $\operatorname{Spec} A$ is open [The20, Tag 01UA] and contains the image of the generic point. Thus, after inverting an element of $A$, we may assume $c$ does not 
vanish along any fiber. In other words, for any $Q \in \operatorname{Spec} A$ and $x \in \operatorname{Spec} R_{k(Q)^{1 / p^{d}}}$, the image of $c$ in $R_{k(Q)}$ is non-zero, and hence also in $R_{k(Q)^{1 / p^{d}}, x}$.

Applying _ $\otimes_{A^{1 / p}} k(Q)^{1 / p^{d+1}}$ to the sequences above gives that

$$
\begin{aligned}
& \left(R_{k(Q)^{1 / p^{d+1}}}\right)^{\oplus p^{\delta}} \rightarrow R_{k(Q)^{1 / p^{d+1}}}^{1 / p} \rightarrow M_{1} \otimes_{A^{1 / p}} k(Q)^{1 / p^{d+1}} \rightarrow 0 \\
& R_{k(Q)^{1 / p^{d+1}}}^{1 / p} \rightarrow\left(R_{k(Q)^{1 / p^{d+1}}}\right)^{\oplus p^{\delta}} \rightarrow M_{2} \otimes_{A^{1 / p}} k(Q)^{1 / p^{d+1}} \rightarrow 0
\end{aligned}
$$

are exact sequences of $R_{k(Q)^{1 / p^{d+1}}}$-modules. But we have that $R_{k(Q)^{1 / p^{d+1}}}$ is a free $R_{k(Q)^{1 / p^{d-}}}$ module of rank $\left[k(Q)^{1 / p}: k(Q)\right]$, so we may view these as sequences of $R_{k(Q)^{1 / p^{d}}}$-modules and localize at $x \in \operatorname{Spec} R_{k(Q)^{1 / p^{d}}}$ to give the exact sequences of $R_{k(Q)^{1 / p^{d}}, x}$-modules

$$
\begin{aligned}
& \left(R_{k(Q)^{1 / p^{d}, x}}\right)^{\oplus p^{\delta}\left[k(Q)^{1 / p}: k(Q)\right]} \stackrel{\psi_{1}}{\longrightarrow}\left(R_{k(Q)^{1 / p^{d}, x}}\right)^{1 / p} \rightarrow\left(M_{1} \otimes_{A^{1 / p}} k(Q)^{1 / p^{d}}\right)_{x}^{\oplus\left[k(Q)^{1 / p}: k(Q)\right]} \rightarrow 0 \\
& \left(R_{k(Q)^{1 / p^{d}, x}}\right)^{1 / p} \stackrel{\psi_{2}}{\longrightarrow}\left(R_{k(Q)^{1 / p^{d}, x}}\right)^{\oplus p^{\delta}\left[k(Q)^{1 / p}: k(Q)\right]} \rightarrow\left(M_{2} \otimes_{A^{1 / p}} k(Q)^{1 / p^{d}}\right)_{x}^{\oplus\left[k(Q)^{1 / p: k(Q)]}\right.} \rightarrow 0
\end{aligned}
$$

so that the summands of the quotients $\left(M_{i} \otimes_{A^{1 / p}} k(Q)^{1 / p^{d}}\right)_{x}$ for $i=1,2$ are killed by the image of $c$ in $R_{k(Q)^{1 / p^{d}, x}}$. If $P$ is the maximal ideal of $R_{k(Q)^{1 / p^{d}, x}}$ and $\ell\left(\_\right)$denotes length over $R_{k(Q)^{1 / p^{d}}, x}$, applying Theorem 4.4 (for $A^{1 / p} \rightarrow R_{A^{1 / p}}$ with the $R_{A^{1 / p} \text {-modules }}$ $M_{1}, M_{2}$ ), we have that there is a positive constant $C^{\prime}$ so that

$\ell\left(\frac{\left(M_{i} \otimes_{A^{1 / p}} k(Q)^{1 / p^{d}}\right)_{x}}{P^{\left[p^{e}\right]}\left(M_{i} \otimes_{A^{1 / p}} k(Q)^{1 / p^{d}}\right)_{x}}\right) \leq \frac{C^{\prime}}{p^{e}} p^{e \cdot \operatorname{dim} R_{k(Q)^{1 / p^{d}},{ }_{, x}}}=\frac{C^{\prime}}{p^{e}} \frac{\left[k(x)^{1 / p}: k(x)\right] p^{(e+1) \operatorname{dim} R_{k(Q)^{1 / p^{d}},{ }_{, x}}}}{\left[k(Q)^{1 / p}: k(Q)\right] p^{\delta}}$

for $i=1,2$ and all $e>0$, and where the last equality follows from setting $e=1$ in the computation (4.6.1).

Let $J_{e}$ denote either the $e$-th Frobenius power $P^{\left[p^{e}\right]}$ of the maximal ideal $P$ used to define the Hilbert-Kunz multiplicity $e_{\mathrm{HK}}\left(R_{k(Q)^{1 / p^{d}, x}}\right)$, or the $e$-th Frobenius degeneracy ideal $I_{e}\left(R_{k(Q)^{1 / p^{d}}, x}\right)$ used to define the $F$-signature. Using the well-known properties $\left(J_{e}\right)^{[p]} \subseteq$ $J_{e+1}$ and $\phi\left(\left(J_{e+1}\right)^{1 / p}\right) \subseteq J_{e}$ for all $\phi \in \operatorname{Hom}_{R_{k(Q)^{1 / p^{d}}, x}}\left(\left(R_{k(Q)^{1 / p^{d}}, x}\right)^{1 / p}, R_{k(Q)^{1 / p^{d}, x}}\right)$. maps $\psi_{1}, \psi_{2}$ induce

$$
\begin{aligned}
& \left(R_{k(Q)^{1 / p^{d}, x}} / J_{e}\right)^{\oplus p^{\delta}\left[k(Q)^{1 / p}: k(Q)\right]} \stackrel{\psi_{1, e}}{\longrightarrow}\left(R_{k(Q)^{1 / p^{d}, x}} / J_{e+1}\right)^{1 / p} \\
& \left(R_{k(Q)^{1 / p^{d}, x}} / J_{e+1}\right)^{1 / p} \stackrel{\psi_{2, e}}{\longrightarrow}\left(R_{k(Q)^{1 / p^{d}, x},} / J_{e}\right)^{\oplus p^{\delta}\left[k(Q)^{1 / p}: k(Q)\right]}
\end{aligned}
$$

with coker $\psi_{i, e}$ a quotient of coker $\psi_{i}$ killed by $P^{\left[p^{e}\right]}$ for $i=1,2$. Taking lengths and dividing by

$$
\left[k(x)^{1 / p}: k(x)\right] p^{(e+1) \operatorname{dim} R_{k(Q)^{1 / p^{d}},{ }_{, x}}}
$$

${ }^{5}$ See for instance [PT18] (and references therein) where these properties are used systematically in the study of Hilbert-Kunz multiplicities and $F$-signatures. 
gives

$$
\left|\frac{\ell\left(\frac{R_{k(Q)^{1 / p^{d}}, x}}{J_{e}}\right)}{p^{e \operatorname{dim} R_{k(Q)^{1 / p^{d}, x}}}}-\frac{\ell\left(\frac{R_{k(Q)^{1 / p^{d}}, x}}{J_{e+1}}\right)}{p^{(e+1) \operatorname{dim} R_{k(Q)^{1 / p^{d}, x}}}}\right| \leq \frac{C^{\prime}}{p^{e}} \cdot \frac{1}{\left[k(Q)^{1 / p}: k(Q)\right] p^{\delta}}
$$

so that the proposition follows from [PT18, Lemma 3.5] with $C=2 C^{\prime} /\left[k(Q)^{1 / p}: k(Q)\right] p^{\delta}$.

As mentioned above, we need to generalize the above to the context of pairs.

Proposition 4.7. Suppose we are in the setting of Setting 4.1. There exists a positive constant $C$ and $0 \neq g \in A$ with the following property: for all $Q \in \operatorname{Spec}\left(B:=A\left[g^{-1}\right]\right)$, all $d>0$, all $x \in \operatorname{Spec}\left(R_{k(Q)^{1 / p^{d}}}\right)$, and all $e>0$, we have

$$
\left|s\left(R_{k(Q)^{1 / p^{d}, x}}, \Delta_{Q, d}\right)-\frac{a_{e}^{\Delta_{Q, d}}\left(R_{k(Q)^{1 / p^{d}, x}}\right)}{\operatorname{rank}_{R_{k(Q)^{1 / p^{d}, x}}}\left(R_{k(Q)^{1 / p^{d}, x}}\right)^{1 / p^{e}}}\right| \leq \frac{C}{p^{e}}
$$

where $\Delta_{Q, d}=\left.\Delta\right|_{\operatorname{Spec}}\left(R_{k(Q)^{1 / p^{d}}}\right) \cdot$

Proof. The desired result follows the argument in Proposition 4.6, with modifications we now describe to account for the addition of $\Delta$. Choose $0 \neq c^{\prime} \in R$ so that $\operatorname{div}_{R}\left(c^{\prime}\right) \geq$ $p \Delta$. After inverting an element of $A$, we may assume $c^{\prime}$ does not vanish along any fiber and thus $\left.\operatorname{div}_{R}\left(c^{\prime}\right)\right|_{\operatorname{Spec} R_{k(Q)}} \geq\left. p \Delta\right|_{\operatorname{Spec} R_{k(Q)}}$ on fibers as well. In particular, for any $\phi \in \operatorname{Hom}_{R_{k(Q)^{1 / p^{d}, x}}}\left(\left(R_{k(Q)^{1 / p^{d}, x}}\right)^{1 / p}, R_{k(Q)^{1 / p^{d}, x}}\right)$ and $\psi\left(\_\right)=\phi\left(\left(c^{\prime}\right)^{1 / p} \cdot{ }_{-}\right)$, we have that $\Delta_{\psi} \geq \Delta_{Q, d}$ and $\left.\operatorname{div}_{R}\left(c^{\prime}\right)\right|_{\operatorname{Spec} R_{k(Q)^{1 / p^{d}}}} \geq p \Delta_{Q, d}$ where $\Delta_{Q, d}=\left.\Delta\right|_{\operatorname{Spec} R_{k(Q)^{1 / p^{d}}}}$.

Replace $\alpha_{1}, \alpha_{2}$ in the right exact sequences 4.6 .2 and 4.6 .3 with their premultiples

$$
\begin{gathered}
\left(R_{A^{1 / p}}\right)^{\oplus p^{\delta}} \stackrel{c^{\prime}}{\longrightarrow}\left(R_{A^{1 / p}}\right)^{\oplus p^{\delta}} \stackrel{\alpha_{1}}{\longrightarrow} R^{1 / p} \\
R^{1 / p} \stackrel{\cdot\left(c^{\prime}\right)^{1 / p}}{\longrightarrow} R^{1 / p} \stackrel{\alpha_{2}}{\longrightarrow}\left(R_{A^{1 / p}}\right)^{\oplus p^{\delta}}
\end{gathered}
$$

respectively. In [PT18, proof of Theorem 4.12], the properties

$$
\begin{gathered}
c^{\prime}\left(I_{e}\left(R_{k(Q)^{1 / p^{d}, x}},\left\lceil\left(p^{e}-1\right) \Delta_{Q, d}\right\rceil\right)^{[p]}\right) \subseteq I_{e+1}\left(R_{k(Q)^{1 / p^{d}}, x},\left\lceil\left(p^{e+1}-1\right) \Delta_{Q, d}\right\rceil\right) \\
\phi\left(\left(c^{\prime} I_{e+1}\left(R_{k(Q)^{1 / p^{d},}, x},\left\lceil\left(p^{e+1}-1\right) \Delta_{Q, d}\right\rceil\right)\right)^{1 / p}\right) \subseteq I_{e}\left(R_{k(Q)^{1 / p^{d}, x}},\left\lceil\left(p^{e}-1\right) \Delta_{Q, d}\right\rceil\right)
\end{gathered}
$$

are shown to hold. The proof of Proposition 4.6 can now be traced through without further modification. The corresponding maps $\psi_{i}$ satisfy the analogs of the above properties with respect to the ideals $I_{e}\left(R_{k(Q)^{1 / p^{d}, x}},\left\lceil\left(p^{e}-1\right) \Delta_{Q, d}\right\rceil\right)$ and pass to maps $\psi_{i, e}$ on the quotients, with coker $\psi_{i, e}$ a quotient of coker $\psi_{i}$ killed by $P^{\left[p^{e}\right]}$ for $i=1,2$. In particular, the constant 
$C^{\prime}$ derived in the proof of Proposition 4.6 from Theorem 4.4 once more gives

$$
\begin{aligned}
& \left|\frac{\ell\left(\frac{R_{k(Q)^{1 / p^{d}}, x}}{I_{e}\left(R_{k(Q)^{1 / p^{d}}, x},\left\lceil\left(p^{e}-1\right) \Delta_{Q, d}\right\rceil\right)}\right)}{p^{e \cdot \operatorname{dim} R_{k(Q)^{1 / p^{d}}, x}}}-\frac{\ell\left(\frac{R_{k(Q)^{1 / p^{d}}, x}}{I_{e+1}\left(R_{k(Q)^{1 / p^{d}}, x},\left\lceil\left(p^{e+1}-1\right) \Delta_{Q, d}\right\rceil\right)}\right)}{p^{(e+1) \operatorname{dim} R_{k(Q)^{1 / p^{d}}, x}}}\right| \\
& \leq \frac{C^{\prime}}{p^{e}} \cdot \frac{1}{\left[k(Q)^{1 / p}: k(Q)\right] p^{\delta}}
\end{aligned}
$$

so that once more the proposition follows from [PT18, Lemma 3.5] with $C=2 C^{\prime} /\left[k(Q)^{1 / p}\right.$ : $k(Q)] p^{\delta}$.

Lemma 4.8. In the setting of Setting 4.1, suppose that there is a surjective $R_{K^{\infty} \text {-linear }}$ map

$$
\left(R_{K^{\infty}}\right)^{1 / p^{e}} \rightarrow R_{K^{\infty}}^{\oplus a_{e}}
$$

for some $a_{e}>0$. Then for some $d_{e}>0$ and $0 \neq g \in A$, setting $B=A[1 / g]$, there is a surjective $R_{B^{1 / p^{e}+d_{e}}}$-linear map

$$
R^{1 / p^{e}} \otimes_{B} B^{1 / p^{e+d_{e}}}=R_{B^{1 / p^{e+d_{e}}}}^{1 / p^{e}} \rightarrow R_{B^{1 / p^{e+d_{e}}}}^{\oplus a_{e}}
$$

which tensors with $\otimes_{B^{1 / p^{e+d}}} K^{\infty}$ to recover (4.8.1).

Furthermore, suppose there exists a Weil divisor $\Delta$ on Spec $R$ (still in Setting 4.1) such that each component projection $\rho: R_{K^{\infty}}^{1 / p^{e}} \rightarrow R_{K^{\infty}}$ corresponds to a $\mathbb{Q}$-divisor $\Delta_{\rho} \geq \xi^{*} \Delta$ (where $\xi: \operatorname{Spec} R_{K^{\infty}} \rightarrow \operatorname{Spec} R$ is the canonical map). In this case we can choose our $g$ such that the map

$$
R_{B^{1 / p^{e+d_{e}}}}^{1 / p^{e}} \rightarrow R_{B^{1 / p^{e+d_{e}}}}^{\oplus a_{e}}
$$

also has the property that each component projection $\gamma: R_{B^{1 / p^{e+d}}}^{1 / d_{e}^{e}} \rightarrow R_{B^{1 / p^{e}+d_{e}}}$ corre-

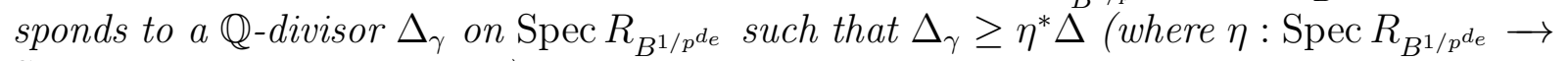
$\operatorname{Spec} R$ is the canonical map).

Proof. First notice since we are planning to invert an element of $A$, we may assume that $\omega_{A} \cong A$. Furthermore, any future $B$ satisfies the same property. Note also that $R_{K^{\infty}}$ is a normal domain by Lemma 2.14. We have $\left(R_{K^{\infty}}\right)^{1 / p^{e}} \cong R^{1 / p^{e}} \otimes_{K^{1 / p^{e}}} K^{\infty}$ and so we can view our initial map as an $R_{K^{\infty}}$-linear map, and in particular a $K^{\infty}$-linear map

$$
\phi:\left(R^{1 / p^{e}}\right)_{K^{\infty}} \rightarrow R_{K^{\infty}}^{\oplus a_{e}}
$$

In other words, we are simply identifying relative and absolute Frobenius over a perfect field. Fix $x_{1}, \ldots, x_{t}$ a generating set for $R^{1 / p^{e}}$ over $R_{A^{1 / p^{e}}}$. By base change, the images of those elements are also a generating set for $R_{K^{\infty}}^{1 / p^{e}}$ over $R_{K^{\infty}}$ or for any intermediate base change. We may assume that all of the $\phi\left(x_{i}\right)$ land inside $R_{K^{1 / p^{e+d_{e}}}}^{\oplus a_{e}} \hookrightarrow R_{K^{\infty}}^{\oplus a_{e}}$ for some

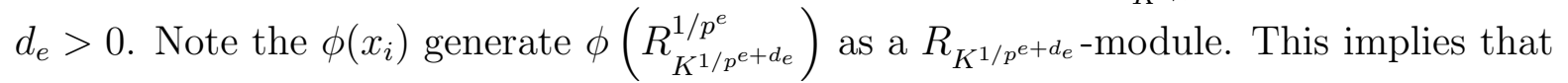

$$
\phi\left(R_{K^{1 / p^{e+d_{e}}}}^{1 / p^{e}}\right) \subseteq R_{K^{1 / p^{e+d_{e}}}}^{\oplus a_{e}}
$$

and hence we have a map (which we also call $\phi$ )

$$
\phi: R_{K^{1 / p^{e+d_{e}}}}^{1 / p^{e}} \rightarrow R_{K^{1 / p^{e+d_{e}}}}^{\oplus a_{e}} .
$$


Since this map becomes surjective after the faithfully flat base change to $K^{\infty}$, it is surjective.

By the same argument as above, we may find a denominator $g^{\prime}$ so that

$$
\phi\left(R_{A^{1 / p^{e+d_{e}}\left[1 / g^{\prime}\right]}}^{1 / p^{e}}\right) \subseteq R_{A^{1 / p^{e+d_{e}}\left[1 / g^{\prime}\right]}}^{\oplus a_{e}}
$$

which produces a map

$$
\phi: R_{A^{1 / p^{e+d_{e}}\left[1 / g^{\prime}\right]}}^{1 / p^{e}} \rightarrow R_{A^{1 / p^{e+d_{e}}\left[1 / g^{\prime}\right]^{\circ}}}^{\oplus a_{e}}
$$

We do not know that this map is surjective but the cokernel is zero if we tensor with $R_{K^{1 / p^{e+d}} e_{e}}$. Inverting another element $g^{\prime \prime}$, setting $g=g^{\prime} g^{\prime \prime}$ and $B=A\left[g^{-1}\right]$, we may assume that

is surjective as desired.

$$
\phi: R_{B^{1 / p^{e+d_{e}}}}^{1 / p^{e}} \rightarrow R_{B^{1 / p^{e+d_{e}}}}^{\oplus a_{e}}
$$

Now we move on to the statement involving $\Delta$. We begin in exactly the same way and produce a surjective map

$$
\phi: R_{B^{1 / p^{e+d_{e}}}}^{1 / p^{e}} \rightarrow R_{B^{1 / p^{e+d_{e}}}}^{\oplus a_{e}}
$$

for some $d_{e}>0$ where $B=A[1 / g]$. We need to show that the component projection maps $\gamma$ coming from $\phi$ produce divisors $\Delta_{\gamma}$ on Spec $R_{B^{1 / p^{d e}}}$ via Section 2.3 such that $\Delta_{\gamma} \geq \eta^{*} \Delta$ where $\eta$ : Spec $R_{B^{1 / p^{d}}} \rightarrow$ Spec $R$ is the canonical map. Consider the following diagram where all of these maps are labeled.

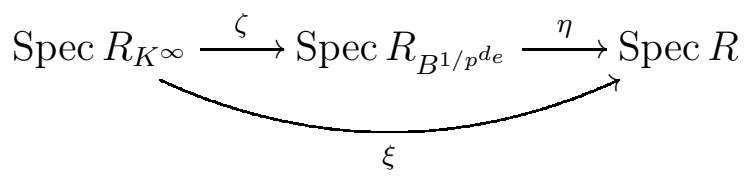

We also know that $\zeta^{*} \Delta_{\gamma}=\Delta_{\rho}$ by Lemma 2.17 since $\gamma$ base changes to a projection $\rho$. Since $\Delta_{\rho} \geq \xi^{*} \Delta=\zeta^{*} \eta^{*} \Delta$, we see that $\zeta^{*} \Delta_{\gamma} \geq \zeta^{*} \eta^{*} \Delta$. Since $\Delta$ has no vertical components neither does $\eta^{*} \Delta$. Therefore because $\Delta_{\gamma} \geq 0$, we conclude that $\Delta_{\gamma} \geq \eta^{*} \Delta$ as desired.

Lemma 4.9. In the setting of Setting 4.1, suppose that there is a surjective $R_{K^{\infty} \text {-linear }}$ map

$$
R_{K^{\infty}}^{\oplus b_{e}} \rightarrow\left(R_{K^{\infty}}\right)^{1 / p^{e}}
$$

for some $b_{e}>0$. Then for some $d_{e}>0$ and $0 \neq g \in A$, setting $B=A[1 / g]$, there is a surjective $R_{B^{1 / p^{e+d}}}$-linear map

$$
R_{B^{1 / p^{e+d_{e}}}}^{\oplus b_{e}} \rightarrow R^{1 / p^{e}} \otimes_{B} B^{1 / p^{e+d_{e}}}=R_{B^{1 / p^{e+d_{e}}}}^{1 / p^{e}}
$$

which tensors with $\otimes_{B^{1 / p^{e+d e}}} K^{\infty}$ to recover (4.9.1).

Proof. The proof strategy is the same as before in Lemma 4.8. Note that $R_{K^{\infty}}$ is a normal domain by Lemma 2.14. We have $\left(R_{K^{\infty}}\right)^{1 / p^{e}} \cong R^{1 / p^{e}} \otimes_{K^{1 / p^{e}}} K^{\infty}$ and so we can view our initial map as an $R_{K^{\infty}}$-linear map, and in particular a $K^{\infty}$-linear map

$$
\psi: R_{K^{\infty}}^{\oplus b_{e}} \rightarrow\left(R^{1 / p^{e}}\right)_{K^{\infty}} .
$$

In other words, we are simply identifying relative and absolute Frobenius over a perfect field. 
The images of the standard basis $e_{i} \in R_{K^{\infty}}^{\oplus b_{e}}$ form a generating set for $\left(R^{1 / p^{e}}\right)_{K^{\infty}}$ by hypothesis. We may assume that $\psi\left(e_{i}\right) \in\left(R^{1 / p^{e}}\right)_{K^{1 / p^{e}+d_{e}}}$ for some $d_{e}>0$. Now, the $\psi\left(e_{i}\right)$ generate $\psi\left(R_{K^{1 / p^{e+d}}}^{\oplus b_{e}}\right)$ as a $R_{K^{1 / p^{e+d}}}$-module and so we have a map which we also call $\psi$

$$
\psi: R_{K^{1 / p^{e+d_{e}}}}^{\oplus b_{e}} \rightarrow\left(R^{1 / p^{e}}\right)_{K^{1 / p^{e+d_{e}}}} .
$$

Since the faithfully flat base change of this map with $K^{\infty}$ is the other map called $\psi$, this $\psi$ is also surjective. Likewise, we also can find a denominator $g^{\prime}$ and so induce a map

$$
\psi: R_{A^{1 / p^{e+d_{e}}}\left[1 / g^{\prime}\right]}^{\oplus b_{e}} \rightarrow\left(R^{1 / p^{e}}\right)_{A^{1 / p^{e+d_{e}}}\left[1 / g^{\prime}\right]} .
$$

Inverting yet another element if necessary, let us assume that this map is also surjective as desired.

Theorem 4.10. In the setting of Setting 4.1, further suppose that $A$ is finite type over a perfect field of characteristic $p>0$. If

$$
s\left(R_{K^{\infty}}, \Delta_{K^{\infty}}\right)>\lambda
$$

then there exists an open dense $U \subseteq \operatorname{Spec} A$ such that for any closed point $Q \in U$,

$$
s\left(R_{k(Q)}, \Delta_{k(Q)}\right)>\lambda .
$$

Proof. Inverting an element of $A$ if necessary, we may choose a positive constant $C$ as in Proposition 4.7. By [DSPY19], fix $0<\epsilon \ll 1$ such that $s\left(R_{K^{\infty}, x}, \Delta_{K^{\infty}}\right)>\lambda+2 \epsilon$ for all $x \in \operatorname{Spec} R_{K^{\infty}}$. Pick $e \gg 0$ so that $C / p^{e}<\epsilon$, so that we have

$$
a_{e}^{\Delta_{K \infty}}\left(R_{K^{\infty}, x}\right) / \operatorname{rank}_{R_{K^{\infty}, x}}\left(R_{K^{\infty}, x}^{1 / p^{e}}\right)>\lambda+\epsilon .
$$

By [DSPY19, Theorem 4.22] and by Lemma 4.8, after inverting an element of $A$ we may assume there is a $d \geq 0$ and a surjective $R_{A^{1 / p^{e+d}}}$-linear map

$$
R_{A^{1 / p^{e+d}}}^{1 / p^{e}} \rightarrow R_{A^{1 / p^{e+d}}}^{\oplus a_{e}}, \text { where } a_{e}:=a_{e}^{\Delta_{K_{\infty}}}\left(R_{K^{\infty}}\right)
$$

satisfying the divisorial condition on projections from Lemma 4.8. Applying $\_\otimes_{A^{1 / p^{e+d}}}$ $k(Q)^{1 / p^{e+d}}$ for maximal $Q \in \operatorname{Spec}(A)$ gives a surjection

$$
R_{k(Q)^{1 / p^{e+d}}}^{1 / p^{e}} \rightarrow R_{k(Q)^{1 / p^{e+d}}}^{\oplus a_{e}}
$$

of $R_{k(Q)^{1 / p^{e+d}}}$-modules where still $a_{e}=a_{e}^{\Delta_{K_{\infty}}}\left(R_{K^{\infty}}\right)$. Note the projections corresponding to this map also have the property that their corresponding divisors are $\geq \Delta_{Q}:=\left.\Delta\right|_{R_{k(Q)^{1 / p^{d}}}}$ by Lemma 2.17.

Since $A$ is finite type over a perfect field and $Q$ is maximal, $k(Q)$ is also perfect and so $k(Q)^{1 / p^{e+d}}=k(Q)^{1 / p^{e}}=k(Q)$. It also follows that

$$
\operatorname{rank}_{R_{K} \infty}\left(R_{K^{\infty}}^{1 / p^{e}}\right)=\operatorname{rank}_{R_{k(Q)}}\left(R_{k(Q)}^{1 / p^{e}}\right)
$$

since $A \subseteq R$ is flat and of finite type and $A$ is $F$-finite.

Therefore we have a surjection

$$
\left(R_{k(Q), x}\right)^{1 / p^{e}} \rightarrow R_{k(Q), x}^{\oplus a_{e}}
$$

showing that

$$
\frac{a_{e}^{\Delta_{Q}}\left(R_{k(Q), x}\right)}{\operatorname{rank}_{R_{k(Q), x}}\left(R_{k(Q), x}\right)^{1 / p^{e}}}>\lambda+\epsilon .
$$


Thus, it follows once again from Proposition 4.7 that

$$
s\left(R_{k(Q), x}, \Delta_{Q}\right)>\lambda
$$

for all $x \in \operatorname{Spec} R_{k(Q)}$ as desired.

Theorem 4.11. In the setting of Setting 4.1, further suppose that $A$ is finite type over a perfect field of characteristic $p>0$. If

$$
e_{\mathrm{HK}}\left(R_{K^{\infty}}\right)<\lambda
$$

then there exists an open dense $U \subseteq \operatorname{Spec} A$ such that for any closed point $Q \in U$,

$$
e_{\mathrm{HK}}\left(R_{k(Q)}\right)<\lambda
$$

Proof. Inverting an element of $A$ if necessary, we may choose a positive constant $C$ as in Proposition 4.6. By [DSPY19], fix $0<\epsilon \ll 1$ such that $e_{\mathrm{HK}}\left(R_{K^{\infty}, x}\right)<\lambda+2 \epsilon$ for all $x \in \operatorname{Spec} R_{K^{\infty}}$. Pick $e \gg 0$ so that $C / p^{e}<\epsilon$, so that we have

$$
b_{e}\left(R_{K^{\infty}, x}\right) / \operatorname{rank}_{R_{K^{\infty}, x}}\left(R_{K^{\infty}, x}^{1 / p^{e}}\right)<\lambda+\epsilon .
$$

By Lemma 4.9, after inverting an element of $A$ we may assume there is a $d \geq 0$ and a surjective $R_{A^{1 / p^{e+d}}}$-linear map

$$
R_{A^{1 / p^{e+d}}}^{\oplus b_{e}} \rightarrow R_{A^{1 / p^{e+d}}}^{1 / p^{e}}, \text { where } b_{e}:=b_{e}\left(R_{K^{\infty}}\right) .
$$

Applying _ $\otimes_{A^{1 / p^{e+d}}} k(Q)^{1 / p^{e+d}}$ for maximal $Q \in \operatorname{Spec}(A)$ gives a surjection

$$
R_{k(Q)^{1 / p^{e+d}}}^{\oplus b_{e}} \rightarrow R_{k(Q)^{1 / p^{e+d}}}^{1 / p^{e}}
$$

of $R_{k(Q)^{1 / p^{e+d}}}$-modules.

Since $A$ is of finite type over a perfect field and $Q$ is maximal, $k(Q)$ is also perfect and so $k(Q)^{1 / p^{e+d}}=k(Q)^{1 / p^{e}}=k(Q)$. It also follows that

$$
\operatorname{rank}_{R_{K^{\infty}}}\left(R_{K^{\infty}}^{1 / p^{e}}\right)=\operatorname{rank}_{R_{k(Q)}}\left(R_{k(Q)}^{1 / p^{e}}\right)
$$

since $A \subseteq R$ is flat and of finite type and $A$ is $F$-finite. Therefore we have a surjection

$$
R_{k(Q), x}^{\oplus b_{e}} \rightarrow\left(R_{k(Q), x}\right)^{1 / p^{e}}
$$

showing that

$$
b_{e}\left(R_{k(Q), x}\right) / \operatorname{rank}_{R_{k(Q), x}}\left(R_{k(Q), x}^{1 / p^{e}}\right)<\lambda+\epsilon .
$$

Thus, it follows once again from Proposition 4.6 that

$$
e_{\mathrm{HK}}\left(R_{k(Q), x}\right)<\lambda
$$

for all $x \in \operatorname{Spec} R_{k(Q)}$ as desired. 


\section{Bertini theorems for F-Signature And Hilbert-Kunz Multiplicity}

In this section, we conclude by proving our Bertini theorems for $F$-signature. We first recall the main result of [CGM86] and the very slight generalization to the context of pairs of SZ13.

Suppose $\mathscr{P}$ is a local property for locally Noetherian schemes (respectively pairs $(X, \Delta \geq$ $0)$ ).

(A1) Whenever $\phi: Y \rightarrow Z$ is a flat morphism with regular fibers and $Z$ (resp. $(Z, \Delta)$ ) is $\mathscr{P}$, then $Y$ (resp. $\left.\left(Y, \phi^{*} \Delta\right)\right)$ is $\mathscr{P}$ too.

(A2) Let $\phi: Y \rightarrow S$ be a morphism of finite type where $Y$ is excellent and $S$ is integral with generic point $\eta$. If $Y_{\eta}$ (resp. $\left(Y_{\eta},\left.\Delta\right|_{Y_{\eta}}\right)$ is geometrically $\mathscr{P}$, then there exists an open neighborhood $U$ of $\eta$ in $S$ such that $Y_{s}\left(\operatorname{resp} .\left(Y_{s},\left.\Delta\right|_{Y_{s}}\right)\right)$ is geometrically $\mathscr{P}$ for each $s \in U$.

(A3) $\mathscr{P}$ is open on schemes $X$ (resp. pairs $(X, \Delta)$ ) of finite type over a field.

Theorem 5.1. CGM86, Theorem 1] Let $X$ be a scheme of finite type over an algebraically closed field $k$, let $\phi: X \rightarrow \mathbb{P}_{k}^{n}$ be a morphism with separable generated residue field extensions. Suppose $X$ (resp. $(X, \Delta)$ ) has a property $\mathscr{P}$ satisfying conditions (A1) and (A2). Then there exists a nonempty open subscheme $U$ of $\left(\mathbb{P}_{k}^{n}\right)^{*}$ such that $\phi^{-1}(H)$ has property $\mathscr{P}$ for each hyperplane $H \in U$.

Remark 5.2. In the proof of Theorem 5.1, when using (A2), $S$ is (an open subset) of $\left(\mathbb{P}_{k}^{n}\right)^{*}$ and $\phi^{-1}(s)=Y_{s}$ are fibers that are exactly equal to the hyperplane sections. In particular, one may additionally assume that $S$ is of finite type over an algebraically closed field and we only need to verify (A2) for the closed fibers.

Suppose that $k=\bar{k}$ is uncountable and consider the following weakening of (A2):

(B2) Let $\phi: Y \rightarrow S$ be a morphism of finite type where $S$ is integral of finite type over $k$, with generic point $\eta$. If $Y_{\eta}$ (resp. $\left(Y_{\eta},\left.\Delta\right|_{Y_{\eta}}\right)$ is geometrically $\mathscr{P}$, then for a very general closed point $s \in S$ we have that $Y_{s}$ (resp. $\left.\left(Y_{s},\left.\Delta\right|_{Y_{s}}\right)\right)$ is geometrically $\mathscr{P}$ for each $s \in U$.

If (A1) and (B2) hold for $\mathscr{P}$, then it immediately follows that the weakening of Theorem 5.1 holds for very general hyperplane sections.

Corollary 5.3. CGM86, Corollary 2] Let $k=\bar{k}, V \subseteq \mathbb{P}_{k}^{n}$ be a closed subscheme (resp. and let $\Delta$ be a $\mathbb{Q}$-divisor on $V$ ) and let $\mathscr{P}$ be a local property satisfying (A1).

(a) If $\mathscr{P}$ satisfies (A2), and $V($ resp. $(V, \Delta))$ is $\mathscr{P}$, then the general hyperplane section of $V$ (resp. $(V, \Delta))$ satisfies $\mathscr{P}$.

(b) If $k$ is uncountable, $\mathscr{P}$ satisfies (B2), and $V$ (resp. $(V, \Delta))$ is $\mathscr{P}$, then the very general hyperplane section of $V($ resp. $(V, \Delta))$ satisfies $\mathscr{P}$.

(c) Suppose $\mathscr{P}$ satisfies (A1), (A2) and (A3), and set $\mathscr{P}(V)$ to be the $\mathscr{P}$ locus of $V$ then

$$
\mathscr{P}(V \cap H) \supseteq \mathscr{P}(V) \cap H
$$

for a general hyperplane $H$.

Combining this machinery with our work of the previous sections, we immediately obtain the main results of the paper. We first state the result for $F$-signature. 
Theorem 5.4. Suppose that $\psi: X \rightarrow \mathbb{P}_{k}^{n}$ is a map of varieties over $k=\bar{k}$ with separably generated residue field extensions (for example, a closed embedding) and that $\Delta \geq 0$ is a $\mathbb{Q}$-divisor on $X$. Suppose that $\lambda \geq 0$.

(a) Suppose $s\left(\mathscr{O}_{X, x}, \Delta_{x}\right)>\lambda$ for all closed points $x \in X$. Choose a general hyperplane $H \subseteq \mathbb{P}_{k}^{n}$, and set $Y=\psi^{-1}(H)$. Then

$$
s\left(\mathscr{O}_{Y, y},\left.\Delta_{y}\right|_{Y}\right)>\lambda
$$

for all closed points $y \in Y$.

(b) Suppose $\psi: X \subseteq \mathbb{P}_{k}^{n}$ is a closed embedding. Let $U_{X} \subseteq X$ be the subset of points $x \in X$ such that $s\left(\mathscr{O}_{X, x}, \Delta_{x}\right)>\lambda$. For any hyperplane $H \subseteq \mathbb{P}_{k}^{n}$ let $U_{H \cap X}$ denote the set of points $x \in X \cap H$ such that $s\left(\mathscr{O}_{H, x},\left.\Delta_{x}\right|_{H}\right)>\lambda$. Then for $H$ a general hyperplane in $\mathbb{P}_{k}^{n}$

$$
U_{H \cap X} \supseteq U_{X} \cap H \text {. }
$$

(c) Suppose additionally that $k$ is uncountable, and that $s\left(\mathscr{O}_{X, x}, \Delta_{x}\right) \geq \lambda$ for all closed points $x \in X$. Choose a very general hyperplane $H \subseteq \mathbb{P}_{k}^{n}$, and set $Y=\psi^{-1}(H)$. Then

$$
s\left(\mathscr{O}_{Y, y},\left.\Delta_{y}\right|_{Y}\right) \geq \lambda
$$

for all closed points $y \in Y$.

Proof. For part (a), we consider the condition $\mathscr{P}$ that $s\left(\mathscr{O}_{X, x}, \Delta\right)>\lambda$. We apply Theorem 5.1 using the fact that properties (A1) and (A2) are satisfied by Theorem 3.6 and Theorem 4.10 respectively (see also Remark 5.2).

For part (b), we simply use Corollary 5.3 and use the fact that $s\left(\mathscr{O}_{X, x}, \Delta\right)>\lambda$ is an open condition by semicontinuity [Pol18, [PT18] so that (A3) is satisfied.

Part (c) either follows from (a) by considering a sequence of $\lambda_{i}=\lambda-1 / i$ or alternately can be directly proven via Remark 5.2 by replacing property (A2) with (B2), which was verified in Proposition 4.2.

Theorem 5.5. Suppose that $\psi: X \rightarrow \mathbb{P}_{k}^{n}$ is a map of normal varieties over $k=\bar{k}$ with separably generated residue field extensions (for example, a closed embedding). Suppose that $\lambda \geq 1$.

(a) Suppose $e_{\mathrm{HK}}\left(\mathscr{O}_{X, x}\right)<\lambda$ for all closed points $x \in X$. Choose a general hyperplane $H \subseteq \mathbb{P}_{k}^{n}$, and set $Y=\psi^{-1}(H)$. Then

$$
e_{\mathrm{HK}}\left(\mathscr{O}_{Y, y}\right)<\lambda
$$

for all closed points $y \in Y$.

(b) Suppose $\psi: X \subseteq \mathbb{P}_{k}^{n}$ is a closed embedding. Let $U_{X} \subseteq X$ be the subset of points $x \in X$ such that $e_{\mathrm{HK}}\left(\mathscr{O}_{X, x}\right)<\lambda$. For any hyperplane $H \subseteq \mathbb{P}_{k}^{n}$ let $U_{H \cap X}$ denote the set of points $x \in X \cap H$ such that $e_{\mathrm{HK}}\left(\mathscr{O}_{H, x}\right)<\lambda$. Then for $H$ a general hyperplane in $\mathbb{P}_{k}^{n}$

$$
U_{H \cap X} \supseteq U_{X} \cap H .
$$

(c) Suppose additionally that $k$ is uncountable, and that $e_{\mathrm{HK}}\left(\mathscr{O}_{X, x}\right) \leq \lambda$ for all closed points $x \in X$. Choose a very general hyperplane $H \subseteq \mathbb{P}_{k}^{n}$, and set $Y=\psi^{-1}(H)$. Then

$$
e_{\mathrm{HK}}\left(\mathscr{O}_{Y, y}\right) \leq \lambda
$$

for all closed points $y \in Y$. 
Proof. For part (a), we consider the condition $\mathscr{P}$ that $e_{\mathrm{HK}}\left(\mathscr{O}_{X, x}\right)<\lambda$. We apply Theorem 5.1 using the fact that properties (A1) and (A2) are satisfied by Theorem 3.7 and Theorem 4.11 respectively.

For part (b), we simply use Corollary 5.3 and use the fact that $e_{\mathrm{HK}}\left(\mathscr{O}_{X, x}\right)<\lambda$ is an open condition by semicontinuity [Smi16, PT18] so that (A3) is satisfied.

Part (c) follows from (a) by considering a sequence of $\lambda_{i}=\lambda-1 / i$.

Remark 5.6. Recently, building on the techniques used in this paper, Datta and Simpson [DS20, Theorem 4.1] have shown that the normality hypothesis on $X$ in Theorem 5.5 can be weakened.

Remark 5.7. We expect that the very general hypothesis in (c) above cannot be removed. Indeed, see [Mon98].

\section{REFERENCES}

[AL03] I. M. Aberbach And G. J. Leuschke: The F-signature and strong F-regularity, Math. Res. Lett. 10 (2003), no. 1, 51-56.

[BST12] M. Blickle, K. Schwede, And K. Tucker: F-signature of pairs and the asymptotic behavior of Frobenius splittings, Adv. Math. 231 (2012), no. 6, 3232-3258.

[Byd18] A. Bydlon: Counterexamples to Bertini theorems for test ideals, J. Algebra 501 (2018), $150-165$.

[CGM86] C. Cumino, S. Greco, and M. Manaresi: An axiomatic approach to the second theorem of Bertini, J. Algebra 98 (1986), no. 1, 171-182.

[DS20] R. DatTa AND A. Simpson: Hilbert-Kunz multiplicity of fibers and Bertini theorems, arXiv:1908.04819.

[DSPY19] A. De Stefani, T. Polstra, and Y. Yao: Globalizing F-invariants, Adv. Math. 350 (2019), 359-395.

[Gab04] O. GabBer: Notes on some t-structures, Geometric aspects of Dwork theory. Vol. I, II, Walter de Gruyter GmbH \& Co. KG, Berlin, 2004, pp. 711-734.

[HW02] N. HARA AND K.-I. WATANABE: F-regular and F-pure rings vs. log terminal and log canonical singularities, J. Algebraic Geom. 11 (2002), no. 2, 363-392.

[Har77] R. Hartshorne: Algebraic geometry, Springer-Verlag, New York, 1977, Graduate Texts in Mathematics, No. 52.

[HH94] M. Hochster And C. Huneke: F-regularity, test elements, and smooth base change, Trans. Amer. Math. Soc. 346 (1994), no. 1, 1-62.

[HH95] M. Hochster And C. HunEke: Applications of the existence of big Cohen-Macaulay algebras, Adv. Math. 113 (1995), no. 1, 45-117.

[HR76] M. Hochster And J. L. Roberts: The purity of the Frobenius and local cohomology, Advances in Math. 21 (1976), no. 2, 117-172.

[HL02] C. Huneke And G. J. Leuschke: Two theorems about maximal Cohen-Macaulay modules, Math. Ann. 324 (2002), no. 2, 391-404.

[Kle98] S. L. Kleiman: Bertini and his two fundamental theorems, Rend. Circ. Mat. Palermo (2) Suppl. (1998), no. 55, 9-37, Studies in the history of modern mathematics, III.

[KM98] J. Kollár AND S. Mori: Birational geometry of algebraic varieties, Cambridge Tracts in Mathematics, vol. 134, Cambridge University Press, Cambridge, 1998, With the collaboration of C. H. Clemens and A. Corti, Translated from the 1998 Japanese original.

[Kun69] E. KunZ: Characterizations of regular local rings for characteristic $p$, Amer. J. Math. 91 (1969), 772-784.

[Kun76] E. KUnZ: On Noetherian rings of characteristic p, Amer. J. Math. 98 (1976), no. 4, 999-1013.

[Laz04] R. Lazarsfeld: Positivity in algebraic geometry. II, Ergebnisse der Mathematik und ihrer Grenzgebiete. 3. Folge. A Series of Modern Surveys in Mathematics [Results in Mathematics and Related Areas. 3rd Series. A Series of Modern Surveys in Mathematics], vol. 49, SpringerVerlag, Berlin, 2004, Positivity for vector bundles, and multiplier ideals. 
[Mat80] H. Matsumura: Commutative algebra, second ed., Mathematics Lecture Note Series, vol. 56, Benjamin/Cummings Publishing Co., Inc., Reading, Mass., 1980.

[Mat89] H. Matsumura: Commutative ring theory, second ed., Cambridge Studies in Advanced Mathematics, vol. 8, Cambridge University Press, Cambridge, 1989, Translated from the Japanese by M. Reid.

[Mon83] P. Monsky: The Hilbert-Kunz function, Math. Ann. 263 (1983), no. 1, 43-49.

[Mon98] P. Monsky: Hilbert-Kunz functions in a family: point-S $_{4}$ quartics, J. Algebra 208 (1998), no. 1, 343-358.

[PSZ18] Z. Patakfalvi, K. Schwede, and W. Zhang: F-singularities in families, Algebr. Geom. 5 (2018), no. 3, 264-327.

[PTY20] F. PÉREz, K. TUCKER, AND Y. YAO: Uniformity in reduction to characteristic $p$, in preparation.

[Pol18] T. Polstra: Uniform bounds in F-finite rings and lower semi-continuity of the F-signature, Trans. Amer. Math. Soc. 370 (2018), no. 5, 3147-3169.

[PT18] T. POLSTRA AND K. TUCKER: F-signature and Hilbert-Kunz multiplicity: a combined approach and comparison, Algebra Number Theory 12 (2018), no. 1, 61-97.

[SZ13] K. Schwede And W. Zhang: Bertini theorems for F-singularities, Proc. Lond. Math. Soc. (3) 107 (2013), no. 4, 851-874.

[Smi16] I. SmiRnov: Upper semi-continuity of the Hilbert-Kunz multiplicity, Compos. Math. 152 (2016), no. 3, 477-488.

[The20] The Stacks Project Authors: stacks project, 2020.

[Tuc12] K. TuCKER: F-signature exists, Invent. Math. 190 (2012), no. 3, 743-765.

[WY00] K.-I. WATANABE AND K.-I. Yoshida: Hilbert-Kunz multiplicity and an inequality between multiplicity and colength, J. Algebra 230 (2000), no. 1, 295-317.

[Yao05] Y. YAO: Modules with finite F-representation type, J. London Math. Soc. (2) 72 (2005), no. 1, $53-72$.

[Yao06] Y. YAO: Observations on the F-signature of local rings of characteristic p, J. Algebra 299 (2006), no. 1, 198-218.

École Polytechnique Fédérale de lausanne, SB math CAG, MA C3 615 (BÂtiment Ma), Station 8, CH-1015 Lausanne, Switzerland

Escuela de Matemática, Universidad de Costa Rica, San José 11501, Costa Rica

Email address: javier.carvajalrojas@epfl.ch

Department of Mathematics, University of Utah, Salt Lake City, UT 84112

Email address: schwede@math.utah.edu

Department of Mathematics, University of Illinois at Chicago, Chicago, IL 60607

Email address: kftucker@uic.edu 